\title{
EXISTENCE FOR VISCOPLASTIC CONTACT WITH COULOMB FRICTION PROBLEMS
}

\author{
AMINA AMASSAD and CAROLINE FABRE
}

Received 19 October 2001

\begin{abstract}
We present existence results in the study of nonlinear problem of frictional contact between an elastic-viscoplastic body and a rigid obstacle. We model the frictional contact both by a Tresca's friction law and a regularized Coulomb's law. We assume, in a first part, that the contact is bilateral and that no separation takes place. In a second part, we consider the Signorini unilateral contact conditions. Proofs are based on a time-discretization method, Banach and Schauder fixed point theorems.
\end{abstract}

2000 Mathematics Subject Classification: 74A55, 74B20, 35Q99, 49J40.

1. Introduction, notation, and main results. This paper deals with the analysis of nonlinear frictional contact problems between an elastic-viscoplastic body and a rigid obstacle. We present both cases of a bilateral contact between the two bodies and a unilateral contact (involving Signorini model) and we consider nonlinear friction law. Before stating the scientific context and our results, we first introduce some notation that will be used in the paper.

Let $\Omega$ be a bounded and regular open set of $\mathbb{R}^{d}$ with boundary $\Gamma$. We suppose that $\Gamma$ is divided in three disjoint parts $\Gamma=\Gamma_{1} \cup \Gamma_{2} \cup \Gamma_{3}$, with $\Gamma_{1}$ being on nonzero measure. We denote by $S_{d}$ ( $d=2$ or 3 ) the space of symmetric tensors of order $d$ on $\mathbb{R}^{d}$ and it is endowed with its natural scalar product.

If $v$ is the unit exterior normal on the boundary $\Gamma$, and if $v$ is a vector in $\mathbb{R}^{d}$, we write $v_{v}=v \cdot v$ and $v_{\tau}=v-v_{v} v$ the normal and tangential decomposition of the vector $v$. In a same way, we write $\sigma_{v}=\sigma v \cdot v$ and $\sigma_{\tau}=\sigma v-\sigma_{v} v$ the normal and tangential components of the vector $\sigma v$ for a tensor $\sigma$.

We consider the following spaces (repeated convention indexes is used):

$$
\begin{gathered}
H=\left[L^{2}(\Omega)\right]^{d}, \\
\mathscr{H}=\left\{\left(\sigma_{i j}\right) \mid \sigma_{i j}=\sigma_{j i} \in L^{2}(\Omega)\right\}=\left[L^{2}(\Omega)\right]_{s}^{d^{2}}, \\
H_{1}=\left[H^{1}(\Omega)\right]^{d}, \\
\mathscr{H}_{1}=H(\operatorname{Div}, \Omega)=\left\{\sigma \in \mathscr{H} \mid\left(\sigma_{i j, j}\right) \in H\right\} .
\end{gathered}
$$

All these spaces are endowed with their natural norms and scalar products as follows: 


$$
\begin{gathered}
(u, v)_{H}=\int_{\Omega} u_{i} v_{i} d x, \quad(\sigma, \tau)_{\mathscr{H}}=\int_{\Omega} \sigma_{i j} \tau_{i j} d x, \\
((u, v))_{H_{1}}=(u, v)_{H}+(\varepsilon(u), \varepsilon(v))_{\mathscr{H}}, \\
((\sigma, \tau))_{\mathscr{H}_{1}}=(\sigma, \tau)_{\mathscr{H}}+(\operatorname{Div} \sigma, \operatorname{Div} \tau)_{H} .
\end{gathered}
$$

When no confusion can be made, we omit the index in the writing of these scalar products.

The notation $\langle,\rangle_{X^{\prime}, X}$ always denotes the duality bracket between a space $X$ and its dual $X^{\prime}$.

We recall the Green formula (valid in regular cases)

$$
(\sigma, \varepsilon(v))_{\mathscr{H}}+(\operatorname{Div} \sigma, v)_{H}=\langle\sigma v, v\rangle_{H^{-1 / 2}(\Gamma)^{d}, H^{1 / 2}(\Gamma) d} \quad \forall v \in H_{1},
$$

which allows to define $\sigma v \in H^{-1 / 2}(\Gamma)^{d}$ for $\sigma \in \mathscr{H}_{1}$ in order that the Green formula still holds.

We consider a Coulomb friction law and in fact a regularization of it in order that the boundary terms in the formulation of our problem make sense. In the sequel $R$ will represent a normal regularization operator that is a linear and continuous operator $R: H^{-1 / 2}(\Gamma) \rightarrow L^{2}(\Gamma)$. We will need it to regularize the trace of the stress tensor on $\Gamma$. Notice that we do not make any hypothesis on compactness property of the operator $R$.

The setting of our problem is as follows: an elastic-viscoplastic body occupies the domain $\Omega$ and is acted upon by given forces and tractions. The body is clamped on $\Gamma_{1} \times(0, T)$ and surface tractions $\varphi_{2}$ act on $\Gamma_{2} \times(0, T)$. The solid is in frictional contact with a rigid obstacle on $\Gamma_{3} \times(0, T)$ and this is where our main interest lies. Moreover, a volume force of density $\varphi_{1}$ acts on the body in $\Omega \times(0, T)$.

We assume a quasistatic process and we consider successively bilateral and unilateral contact. The unilateral case is modelled by Signorini's contact condition. With these assumptions, the mechanical problem of frictional contact of the viscoplastic body may be formulated as follows (see [5, 6, 7]).

Bilateral Contact. Find a displacement field $u: \Omega \times[0, T] \rightarrow \mathbb{R}^{d}$ and a stress field $\sigma: \Omega \times[0, T] \rightarrow S_{d}$ such that

$$
\begin{aligned}
& \dot{\sigma}=\mathscr{E} \varepsilon(\dot{u})+G(\sigma, \varepsilon(u)) \quad \text { in } \Omega \times(0, T), \\
& \operatorname{Div} \sigma+\varphi_{1}=0 \quad \text { in } \Omega \times(0, T), \\
& \begin{array}{l}
u=0 \quad \text { on } \Gamma_{1} \times(0, T), \\
\sigma v=\varphi_{2} \quad \text { on } \Gamma_{2} \times(0, T), \\
u_{v}=0, \quad\left|\sigma_{\tau}\right| \leq \mu\left|R\left(\sigma_{v}\right)\right| \quad \text { on } \Gamma_{3} \times(0, T), \\
\text { with }\left\{\begin{array}{l}
\left|\sigma_{\tau}\right|<\mu\left|R\left(\sigma_{v}\right)\right| \Longrightarrow \dot{u}_{\tau}=0, \\
\left|\sigma_{\tau}\right|=\mu\left|R\left(\sigma_{v}\right)\right| \Longrightarrow \exists \lambda \geq 0 \quad \text { such that } \sigma_{\tau}=-\lambda \dot{u}_{\tau},
\end{array}\right. \\
u(0)=u^{0}, \quad \sigma(0)=\sigma^{0} \quad \text { in } \Omega .
\end{array}
\end{aligned}
$$


UNilateral contact. Find a displacement field $u: \Omega \times[0, T] \rightarrow \mathbb{R}^{d}$ and a stress field $\sigma: \Omega \times[0, T] \rightarrow S_{d}$ such that

$$
\begin{aligned}
& \dot{\sigma}=\mathscr{E} \varepsilon(\dot{u})+G(\sigma, \varepsilon(u)) \quad \text { in } \Omega \times(0, T), \\
& \operatorname{Div} \sigma+\varphi_{1}=0 \quad \text { in } \Omega \times(0, T), \\
& u=0 \quad \text { on } \Gamma_{1} \times(0, T), \\
& \sigma v=\varphi_{2} \quad \text { on } \Gamma_{2} \times(0, T), \\
& u_{v} \leq 0, \quad \sigma_{v} \leq 0, \quad u_{v} \sigma_{v}=0 \quad \text { on } \Gamma_{3} \times(0, T), \\
& \left|\sigma_{\tau}\right| \leq \mu\left|R\left(\sigma_{v}\right)\right| \quad \text { on } \Gamma_{3} \times(0, T), \\
& \text { with }\left\{\begin{array}{l}
\left|\sigma_{\tau}\right|<\mu\left|R\left(\sigma_{v}\right)\right| \Longrightarrow \dot{u}_{\tau}=0, \\
\left|\sigma_{\tau}\right|=\mu\left|R\left(\sigma_{v}\right)\right| \Longrightarrow \exists \lambda \geq 0 \quad \text { such that } \sigma_{\tau}=-\lambda \dot{u}_{\tau},
\end{array}\right. \\
& u(0)=u^{0}, \quad \sigma(0)=\sigma^{0} \quad \text { in } \Omega .
\end{aligned}
$$

We now state hypotheses on the data involved in the constitutive law and forces.

Hypotheses on the tensor $\mathscr{E}: \mathscr{E}: \Omega \times S_{d} \rightarrow S_{d}$ is a symmetric definite positive tensor which means

$$
\begin{gathered}
\mathscr{E}_{i j k l} \in L^{\infty}(\Omega), \quad \forall i, j, k, l=1, \ldots, d, \\
\mathscr{E} \sigma \cdot \tau=\sigma \cdot \mathscr{E} \tau, \quad \forall \sigma, \tau \in S_{d}, \text { a.e. in } \Omega, \\
\exists \alpha>0 \quad \text { such that } \mathscr{E} \sigma \cdot \sigma \geq \alpha|\sigma|^{2}, \quad \forall \sigma \in S_{d} .
\end{gathered}
$$

Hypotheses on the nonlinearity $G$ :

$$
G: \Omega \times S_{d} \times S_{d} \rightarrow S_{d}
$$

provided that

$$
\begin{aligned}
& \exists L>0 \text { such that } \\
& \begin{array}{l}
\left|G\left(x, \sigma_{1}, \varepsilon_{1}\right)-G\left(x, \sigma_{2}, \varepsilon_{2}\right)\right| \leq L\left(\left|\sigma_{1}-\sigma_{2}\right|+\left|\varepsilon_{1}-\varepsilon_{2}\right|\right), \\
\forall \forall \sigma_{1}, \sigma_{2}, \varepsilon_{1}, \varepsilon_{2} \in S_{d}, \quad \text { a.e. in } \Omega,
\end{array} \\
& x \longmapsto G(x, \sigma, \varepsilon) \quad \text { is Lebesgue measurable on } \Omega, \forall \sigma, \varepsilon \in S_{d}, \\
& x \longmapsto G(x, 0,0) \in \mathcal{H} .
\end{aligned}
$$

Hypotheses on forces

$$
\varphi_{1} \in H^{1}(0, T ; H), \quad \varphi_{2} \in H^{1}\left(0, T ; L^{2}\left(\Gamma_{2}\right)^{d}\right) .
$$

These hypotheses make sense to the definition of an element $f \in H^{1}\left(0, T ; V^{\prime}\right)$ with $\left(\gamma_{0} v\right.$ is the trace over $\Gamma$ of the vector $v$ )

$$
\langle f(t), v\rangle_{V^{\prime}, V}=\int_{\Omega} \varphi_{1}(t) v+\left(\varphi_{2}(t), \gamma_{0} v\right)_{L^{2}\left(\Gamma_{2}\right)} d
$$

In order to prove existence results concerning these problems, we first give equivalent variational formulations and to this aim, we need to introduce the following spaces:

$$
V=\left\{v \in H_{1} \mid v=0 \text { on } \Gamma_{1}\right\},
$$


and $V_{0}, K$ are subspace and convex set of admissible displacements, respectively (both nonempty and closed in $V$ ):

$$
\begin{gathered}
V_{0}=V \cap\left\{v \in H_{1} \mid v_{v}=0 \text { on } \Gamma_{3}\right\}, \\
K=V \cap\left\{v \in H_{1} \mid v_{v} \leq 0 \text { on } \Gamma_{3}\right\} .
\end{gathered}
$$

We recall that Korn's inequality leads to the following statement on $V$ (see [5]):

$$
|\varepsilon(u)|_{\mathscr{H}} \geq C\|u\|_{V}, \quad \forall u \in V
$$

which, with (1.6), implies that $v \rightarrow \sqrt{a(v, v)}$ with

$$
a(v, v)=\int_{\Omega} \mathscr{E} \varepsilon(v)(x) \cdot \varepsilon(v)(x) d x
$$

defines an equivalent norm on $V$.

If a function $g=g(t, a)$ is in $L^{2}\left(0, T ; L^{2}\left(\Gamma_{3}\right)\right)$, we introduce the functional

$$
j(g(t), v)=\mu \int_{\Gamma_{3}}|g(t, a)|\left|v_{\tau}(a)\right| d a,
$$

where $d a$ is the surface measure on $\Gamma_{3}$.

We can now state the variational formulations of problems (1.4) and (1.5).

Bilateral contact. Find $u:[0, T] \rightarrow V_{0}$ and $\sigma:[0, T] \rightarrow \mathscr{H}_{1}$ with

$$
\begin{aligned}
& \dot{\sigma}(t)=\mathscr{E} \varepsilon(\dot{u}(t))+G(\sigma(t), \varepsilon(u(t))), \quad \text { a.e. } t \in(0, T), \\
& (\sigma(t), \varepsilon(v)-\varepsilon(\dot{u}(t)))_{\mathscr{H}}+j\left(R\left(\sigma_{v}\right)(t), v\right)-j\left(R\left(\sigma_{v}\right)(t), \dot{u}(t)\right) \\
& \geq\langle f(t), v-\dot{u}(t)\rangle_{V^{\prime}, V}, \quad \forall v \in V_{0}, \text { a.e. } t \in(0, T), \\
& u(0)=u^{0}, \quad \sigma(0)=\sigma^{0} .
\end{aligned}
$$

It is known that we cannot expect existence of a solution without a compatibility condition between $u^{0}, \sigma^{0}$, and $f(0)$. In the bilateral contact case, we will suppose that

$$
\begin{gathered}
u^{0} \in V_{0}, \quad \sigma^{0} \in \mathscr{H}_{1}, \\
\left(\sigma^{0}, \varepsilon(v)\right)_{\mathscr{H}}+j\left(R\left(\sigma_{v}^{0}\right), v\right) \geq\langle f(0), v\rangle_{V^{\prime}, V}, \quad \forall v \in V_{0} .
\end{gathered}
$$

UNILATERAL CONTACT. Following Cocu et al. [4] for the formulation of the unilateral contact condition, we introduce the space $H\left(\Gamma_{3}\right)$ as the set of restrictions to $\Gamma_{3}$ of the $H^{1 / 2}(\Gamma)$ functions which are null on $\Gamma_{1}$. For every $\sigma \in \mathscr{H}_{1}$, let $\langle\cdot, \cdot\rangle$ denote the duality pairing between $H\left(\Gamma_{3}\right)$ and its dual with

$$
\left\langle\sigma_{v}, v_{v}\right\rangle=\int_{\Gamma_{3}} \sigma_{v}(a) v_{v}(a) d a, \quad \forall v \in V
$$


in regular situations (and which involves the Green formula in nonregular ones). The formulation is then as follows: find $u:[0, T] \rightarrow K$ and $\sigma:[0, T] \rightarrow \mathscr{H}_{1}$ with

$$
\begin{aligned}
& \dot{\sigma}(t)=\mathscr{E} \varepsilon(\dot{u}(t))+G(\sigma(t), \varepsilon(u(t))), \quad \text { a.e. } t \in(0, T), \\
& (\sigma(t), \varepsilon(v)-\varepsilon(\dot{u}(t)))_{\mathscr{H}}+j\left(R\left(\sigma_{v}\right)(t), v\right)-j\left(R\left(\sigma_{v}\right)(t), \dot{u}(t)\right) \\
& \quad \geq\langle f(t), v-\dot{u}(t)\rangle_{V^{\prime}, V}+\left\langle\sigma_{v}(t), v_{v}-\dot{u}_{v}(t)\right\rangle, \quad \forall v \in V, \text { a.e. } t \in(0, T), \\
& \left\langle\sigma_{v}(t), v_{v}-u_{v}(t)\right\rangle \geq 0, \quad \forall v \in K, \\
& u(0)=u^{0}, \quad \sigma(0)=\sigma^{0} .
\end{aligned}
$$

The compatibility condition between the data is now

$$
\begin{gathered}
u^{0} \in K, \quad \sigma^{0} \in \mathscr{H}_{1}, \\
\left(\sigma^{0}, \varepsilon(v)\right)_{\mathscr{H}}+j\left(R\left(\sigma_{v}^{0}\right), v\right) \geq\langle f(0), v\rangle_{V^{\prime}, V}+\left\langle\sigma_{v}^{0}, v_{v}\right\rangle, \quad \forall v \in V, \\
\left\langle\sigma_{v}^{0}, v_{v}-u_{v}^{0}\right\rangle \geq 0, \quad \forall v \in K .
\end{gathered}
$$

We prove the following theorem.

Theorem 1.1 (bilateral case). Suppose that (1.6), (1.8), (1.9), and (1.17) hold. Then, there exists $\mu_{0}>0$, such that for $0<\mu \leq \mu_{0}$, there exists at least one solution, $(u, \sigma)$ with $u \in H^{1}\left(0, T ; V_{0}\right)$ and $\sigma \in H^{1}\left(0, T ; \mathcal{H}_{1}\right)$, to problem (1.16). Furthermore, if the given forces $\varphi_{1}$ and $\varphi_{2}$ are in $W^{1, \infty}(0, T ; H)$ and $W^{1, \infty}\left(0, T ; L^{2}\left(\Gamma_{2}\right)^{d}\right)$, then $(u, \sigma) \in$ $W^{1, \infty}\left(0, T ; V_{0}\right) \times W^{1, \infty}\left(0, T ; \mathscr{H}_{1}\right)$.

Theorem 1.2 (unilateral case). Suppose that (1.6), (1.8), (1.9), and (1.20) hold. Then, there exists $\mu_{0}>0$, such that for $0<\mu \leq \mu_{0}$, there exists at least one solution, $(u, \sigma)$ with $u \in H^{1}(0, T ; V) \cap C([0, T] ; K)$ and $\sigma \in H^{1}\left(0, T ; \mathscr{H}_{1}\right)$, to problem (1.19). Furthermore, if the given forces $\varphi_{1}$ and $\varphi_{2}$ are in $W^{1, \infty}(0, T ; H)$ and $W^{1, \infty}\left(0, T ; L^{2}\left(\Gamma_{2}\right)^{d}\right)$, then $(u, \sigma) \in$ $W^{1, \infty}(0, T ; V) \times W^{1, \infty}\left(0, T ; \mathscr{H}_{1}\right)$.

Theorem 1.2 was obtained by Cocu et al. in [4] when the constitutive law is an elastic one $(\sigma=\mathscr{E} \varepsilon(u))$ and for a compact operator $R$. We recall that here, no such hypothesis is made; the main meaning of this is that our friction law could be a local one on any part of the contact zone where the term $\sigma_{v}$ has a sense almost everywhere.

Considering now viscoelastic constitutive law, $\sigma=\mathscr{E}(\varepsilon(\dot{u}))+G(\varepsilon(u))$ with Lipschitz functions $\mathscr{E}$ (still coercive) and $G$, existence results for bilateral contact was proved by Shillor and Sofonea in [8] and announced (with a sketch of the proof) by the authors in [1] in case of unilateral contact.

Concerning uniqueness, we can read a paper of Ballard [3] where existence results are given for the cinematic of a material point and where a counter example to uniqueness is given. However no counter example have been exhibit in the PDE's framework.

Finally, we mention [2] by Amassad and Sofonea where existence and uniqueness results are proved for viscoplastic constitutive law when the friction is modelled with a Tresca law involving a constant friction coefficient. 
We end this section detailing our plan: we present different proofs for bilateral and Signorini contact formulations. In case of bilateral contact, we use a fixed point method: we first study the case of friction Tresca's law with a friction coefficient $g$ (instead of $R\left(\sigma_{v}\right)$ ) depending on the time variable. The fixed point will then involve the map $g \rightarrow R\left(\sigma_{v}\right)$. In case of unilateral contact of Signorini, the fixed point method which was used before is no more valid. Indeed, the main point is that we can no more define a map $g \rightarrow R\left(\sigma_{v}\right)$. This relation could just allow to define a multivalued map for which we did not find a fixed point theorem that may be applied, and the reason is a lack of convexity. This is the theoretical main difference between the two contact conditions. In Section 2, we present the study of bilateral contact with Tresca friction law. In Section 3, we prove Theorem 1.1 in bilateral case whereas the unilateral case given in Theorem 1.2 is proved in Section 4.

2. Bilateral contact and Tresca's law. In this section, we are interested in Tresca's friction law when the friction yield limit may depend on the time variable. Our motivation for this is a fixed point method which will be used in Section 3.

The friction process is modelled with a function $g=g(x, t)$ defined on $\Gamma_{3} \times(0, T)$ with

$$
g \in H^{1}\left(0, T ; L^{2}\left(\Gamma_{3}\right)\right)
$$

We will also study the case where $g \in W^{1, \infty}\left(0, T ; L^{2}\left(\Gamma_{3}\right)\right)$.

Initial data are supposed to verify the following compatibility condition:

$$
\begin{gathered}
u^{0} \in V_{0}, \quad \sigma^{0} \in \mathscr{H}_{1}, \\
\left(\sigma^{0}, \varepsilon(v)\right)_{\mathscr{H}}+j(g(0), v) \geq\langle f(0), v\rangle_{V^{\prime}, V}, \quad \forall v \in V_{0} .
\end{gathered}
$$

The model can then be written as

$$
\begin{aligned}
& \dot{\sigma}=\mathscr{E} \varepsilon(\dot{u})+G(\sigma, \varepsilon(u)) \quad \text { in } \Omega \times(0, T), \\
& \operatorname{Div} \sigma+\varphi_{1}=0 \quad \text { in } \Omega \times(0, T), \\
& u=0 \quad \text { on } \Gamma_{1} \times(0, T), \\
& \sigma v=\varphi_{2} \quad \text { on } \Gamma_{2} \times(0, T), \\
& u_{v}=0, \quad\left|\sigma_{\tau}\right| \leq \mu|g(t)| \quad \text { on } \Gamma_{3} \times(0, T), \\
& \text { with }\left\{\begin{array}{l}
\left|\sigma_{\tau}\right|<\mu|g(t)| \Longrightarrow \dot{u}_{\tau}=0, \\
\left|\sigma_{\tau}\right|=\mu|g(t)| \Longrightarrow \exists \lambda \geq 0 \quad \text { such that } \sigma_{\tau}=-\lambda \dot{u}_{\tau},
\end{array}\right. \\
& u(0)=u^{0}, \quad \sigma(0)=\sigma^{0} \quad \text { in } \Omega,
\end{aligned}
$$


and its variational formulation is given by

$$
\begin{aligned}
\dot{\sigma}(t) & =\mathscr{E} \varepsilon(\dot{u}(t))+G(\sigma(t), \varepsilon(u(t))), \quad \text { a.e. } t \in(0, T), \\
(\sigma(t), & \varepsilon(v)-\varepsilon(\dot{u}(t)))_{\mathscr{H}}+j(g(t), v)-j(g(t), \dot{u}(t)) \\
& \geq\langle f(t), v-\dot{u}(t)\rangle_{V^{\prime}, V}, \quad \forall v \in V_{0}, \text { a.e. } t \in(0, T), \\
u(0) & =u^{0}, \quad \sigma(0)=\sigma^{0} .
\end{aligned}
$$

We prove the following proposition.

Proposition 2.1. Suppose that (1.6), (1.8), (1.9), (2.1), and (2.2) hold. Then there exists a unique solution $(u, \sigma)$ to problem (2.4) and

$$
u \in H^{1}\left(0, T ; V_{0}\right), \quad \sigma \in H^{1}\left(0, T ; \mathscr{H}_{1}\right) .
$$

Moreover, if $g \in W^{1, \infty}\left(0, T ; L^{2}\left(\Gamma_{3}\right)\right)$, the solution is in $W^{1, \infty}\left(0, T ; V_{0}\right) \times W^{1, \infty}\left(0, T ; \mathscr{H}_{1}\right)$.

Notice that Proposition 2.1 gives existence and uniqueness of the solution.

The proof of Proposition 2.1 is based on a time discretization method when the plasticity map $G$ is supposed to be null (Section 2.1) followed by a Banach fixed point (see Section 2.2). This kind of proof is rather classical and has been used in [2] where it was supposed that the friction coefficient $g=g(x)$ was constant with respect to the time variable.

2.1. Existence and uniqueness in the elastic case. In this section, we suppose that the function $G$ is null and that $\sigma^{0}=\mathscr{E} \varepsilon\left(u^{0}\right)$ which leads to the elastic constitutive law $\sigma(t)=\mathscr{E} \varepsilon(u(t))$.

We suppose that (1.6), (1.8), (1.9), (2.1), and (2.2) hold and we are interested in the formulation

$$
\begin{aligned}
& \begin{aligned}
\sigma(t) & =\mathscr{E} \varepsilon(u(t)), \quad \text { a.e. } t \in(0, T), \\
(\sigma(t), & \varepsilon(v)-\varepsilon(\dot{u}(t)))_{\mathscr{H}}+j(g(t), v)-j(g(t), \dot{u}(t)) \\
& \geq\langle f(t), v-\dot{u}(t)\rangle_{V^{\prime}, V}, \quad \forall v \in V_{0}, \text { a.e. } t \in(0, T),
\end{aligned} \\
& u(0)=u^{0}
\end{aligned}
$$

For simplicity, we denote by $\|\cdot\|\left(\right.$ resp., $\left.|\cdot|_{3}\right)$ the $H^{1}\left(0, T ; V^{\prime}\right)$-norm (resp., the $L^{2}(0, T$; $\left.L^{2}\left(\Gamma_{3}\right)\right)$-norm).

We prove the following proposition.

Proposition 2.2. Under hypotheses (1.6), (1.8), (1.9), (2.1), and (2.2), there exists a unique solution to problem (2.6) having the regularity $u \in H^{1}\left(0, T ; V_{0}\right)$. Moreover, there exists a constant $c>0$, such that for every $u^{0}, f$, and $g$ satisfying, respectively 
(2.2), (1.9), and (2.1), we have

$$
|u|_{H^{1}\left(0, T ; V_{0}\right)} \leq c\left[|g|_{3}+\|f\|+\left|u^{0}\right|_{V}\right] .
$$

If $g \in W^{1, \infty}\left(0, T ; L^{2}\left(\Gamma_{3}\right)\right)$ and $f \in W^{1, \infty}\left(0, T ; V^{\prime}\right)$, then $u \in W^{1, \infty}\left(0, T ; V_{0}\right)$ and

$$
|u|_{W^{1, \infty}\left(0, T ; V_{0}\right)} \leq c\left[|g|_{W^{1, \infty}\left(0, T ; L^{2}\left(\Gamma_{3}\right)\right)}+|f|_{W^{1, \infty}\left(0, T ; V^{\prime}\right)}+\left|u^{0}\right|_{V}\right] .
$$

In the case where the friction coefficient $g$ is a positive nondecreasing function with respect to the time variable, we prove the following proposition.

Proposition 2.3. Suppose that for almost every $x \in \Gamma_{3}, t \rightarrow g(t, x)$ is a positive nondecreasing function on ]0,T [, then there exists $c>0$, such that for every $u^{0}, f$ satisfying (1.9) and (2.2), we have

$$
|u|_{H^{1}\left(0, T ; V_{0}\right)} \leq c\left[\|f\|+\left|u^{0}\right|_{V}\right]
$$

Proposition 2.3 adds a uniform bound with respect to the friction coefficient.

Proof of Proposition 2.2. We detail the proof when $g \in W^{1, \infty}\left(0, T ; L^{2}\left(\Gamma_{3}\right)\right)$ and $f \in W^{1, \infty}\left(0, T ; V^{\prime}\right)$ (following it, we will then easily see that the same proof is valid for other cases). We use a time discretization method with respect to the time variable and we construct a sequence of time-continuous functions denoted by $\left(u_{N}\right)_{N}$ in the following way: the integer $N$ being given, we write $h=\Delta t=T / N$, and $t_{n}=n h$ for $0 \leq n \leq N$. Let $X$ be a Banach space and $f$ be a function in $H^{1}(0, T ; X)$. We write $f_{n}=f\left(t_{n}\right)$ and we introduce the following functions

$$
\begin{gathered}
\left.\left.\tilde{f}_{N}(t)=f_{n+1} \quad \text { on }\right] t_{n}, t_{n+1}\right] \\
f_{N}(t)=f_{n}+\left(t-t_{n}\right)\left(\frac{f_{n+1}-f_{n}}{h}\right) \text { on }\left[t_{n}, t_{n+1}\right] .
\end{gathered}
$$

We recall that $\left(f_{N}\right)_{N}$ strongly converges to $f$ in $H^{1}(0, T ; X)$ and $\left(\tilde{f}_{N}\right)_{N}$ strongly converges to $f$ in $L^{2}(0, T ; X)$. These properties will be useful for the forces $f$ and the friction coefficient $g$ (with respectively $X=V^{\prime}$ and $X=L^{2}\left(\Gamma_{3}\right)$ ).

We consider the time-continuous function $u_{N}$ on $(0, T)$ and affine on each interval ]$t_{n}, t_{n+1}[$ defined by

$$
u_{N}(t)=u_{n}+\left(t-t_{n}\right)\left(\frac{u_{n+1}-u_{n}}{h}\right) \text { on }\left[t_{n}, t_{n+1}\right]
$$

where the sequence of values at points $n h,\left(u_{n}\right)_{0 \leq n \leq N}$ is characterized by the following iterative process: $u_{0}=u^{0}$ and $u_{n+1} \in V_{0}$ is solution of the optimization problem

$$
J_{n}\left(u_{n+1}\right)=\min _{w \in V_{0}} J_{n}(w)
$$


where (recall that $g_{n}=g\left(t_{n}\right)$ and $f_{n}=f\left(t_{n}\right)$ )

$$
J_{n}(w)=\frac{1}{2} a(w, w)+\mu \int_{\Gamma_{3}}\left|g_{n+1}\right|\left|w_{\tau}-u_{n \tau}\right| d a-\left\langle f_{n+1}, w\right\rangle_{V^{\prime}, V} .
$$

We write

$$
j_{n+1}(v)=\mu \int_{\Gamma_{3}}\left|g_{n+1}\right|\left|v_{\tau}\right| d a .
$$

We can easily prove existence and uniqueness of the minimizer $u_{n+1} \in V_{0}$ of the functional $J_{n}$. Furthermore, due to the convexity of $J_{n}, u_{n+1}$ satisfies the following inequality:

$$
\begin{aligned}
a\left(u_{n+1}, w-u_{n+1}\right)+ & j_{n+1}\left(w-u_{n}\right)-j_{n+1}\left(u_{n+1}-u_{n}\right) \\
\geq & \left\langle f_{n+1}, w-u_{n+1}\right\rangle_{V^{\prime}, V}, \quad \forall w \in V_{0} .
\end{aligned}
$$

We now prove that $\left(u_{N}\right)_{N}$ is bounded in $W^{1, \infty}(0, T ; V)$. Taking $w=0$ in (2.15), we obtain for $0 \leq n \leq N-1$,

$$
\begin{aligned}
a\left(u_{n+1}, u_{n+1}\right) & \leq\left\langle f_{n+1}, u_{n+1}\right\rangle_{V^{\prime}, V}+j_{n+1}\left(u_{n}\right)-j_{n+1}\left(u_{n+1}-u_{n}\right) \\
& \leq\left\langle f_{n+1}, u_{n+1}\right\rangle_{V^{\prime}, V}+j_{n+1}\left(u_{n+1}\right),
\end{aligned}
$$

hence the sequence $\left(u_{N}\right)_{N}$ is bounded in $C\left([0, T] ; V_{0}\right)$ and

$$
\max _{0 \leq t \leq T}\left\|u_{N}(t)\right\|_{V_{0}} \leq c\left[\|f\|_{C\left([0, T] ; V^{\prime}\right)}+\|g\|_{C\left([0, T] ; L^{2}\left(\Gamma_{3}\right)\right)}\right] .
$$

Now, taking $w=u_{n}$ in inequality (2.15) satisfied by $u_{n+1}$ and $w=u_{n+1}$ in inequality (2.15) satisfied by $u_{n}$, we obtain (using the compatibility condition (2.2) for the first step)

$$
\begin{aligned}
a\left(u_{n+1}-u_{n}, u_{n+1}-u_{n}\right) \leq & \left\langle f_{n+1}-f_{n}, u_{n+1}-u_{n}\right\rangle_{V^{\prime}, V}+j_{n}\left(u_{n+1}-u_{n-1}\right) \\
& -j_{n-1}\left(u_{n}-u_{n-1}\right)-j_{n-1}\left(u_{n+1}-u_{n}\right)
\end{aligned}
$$

and hence (using the definition of $j_{n}$ )

$$
\begin{aligned}
a\left(u_{n+1}-u_{n}, u_{n+1}-u_{n}\right) \leq & \left\langle f_{n+1}-f_{n}, u_{n+1}-u_{n}\right\rangle_{V^{\prime}, V} \\
& +\mu \int_{\Gamma_{3}}\left(\left|g_{n}\right|-\left|g_{n+1}\right|\right)\left|u_{(n+1) \tau}-u_{n \tau}\right| \text { da. }
\end{aligned}
$$

We deduce that the sequence $\left(\dot{u}_{N}\right)_{N}$ is bounded in $L^{\infty}(0, T ; V)$ and that there exists $c>0$ such that for all $\mu>0$, we get

$$
\begin{aligned}
\left\|\dot{u}_{N}\right\|_{L^{\infty}(0, T ; V)} & =\max _{0 \leq n \leq N-1}\left\|\frac{u_{n+1}-u_{n}}{h}\right\|_{V} \\
& \leq c\left[\mu\|\dot{g}\|_{L^{\infty}\left(0, T ; L^{2}\left(\Gamma_{3}\right)\right)}+\|\dot{f}\|_{L^{\infty}\left(0, T ; V^{\prime}\right)}\right] .
\end{aligned}
$$


Then, there exists $u \in W^{1, \infty}\left(0, T ; V_{0}\right)$ such that (after extraction of a subsequence)

$$
u_{N} \longrightarrow u \text { in } W^{1, \infty}(0, T ; V) \text { weak-** }
$$

Remark that this convergence implies that

$$
\forall t \in[0, T], \quad u_{N}(t) \longrightarrow u(t) \quad \text { weakly in } V .
$$

Furthermore, since $\left|u_{N}(t)-\tilde{u}_{N}(t)\right|_{V} \leq h\left|\dot{u}_{N}(t)\right|_{V}$, for every $t \in[0, T]$, the sequence $\left(\tilde{u}_{N}(t)\right)_{N}$ converges weakly in $V$ to $u$.

In order to prove that the vector-valued function $u$ is solution of (2.6), we write inequality (2.15) in another way; using the scaling $w=h v+u_{n}$ and since $u_{n} \in V_{0}$ and $j_{n}(h v)=h j_{n}(v)$ (for every $n$ ), we can easily see that we can write (2.15) in the following equivalent way:

$$
\begin{array}{r}
a\left(u_{n+1}, v-\frac{u_{n+1}-u_{n}}{h}\right)+j_{n+1}(v)-j_{n+1}\left(\frac{u_{n+1}-u_{n}}{h}\right) \\
\geq\left\langle f_{n+1}, v-\frac{u_{n+1}-u_{n}}{h}\right\rangle_{V^{\prime}, V}, \quad \forall v \in V_{0},
\end{array}
$$

or

$$
\begin{aligned}
& a\left(\tilde{u}_{N}(t), v-\dot{u}_{N}(t)\right)+j\left(\tilde{g}_{N}(t), v\right)-j\left(\tilde{g}_{N}(t), \dot{u}_{N}(t)\right) \\
& \left.\left.\geq\left\langle\tilde{f}_{N}(t), v-\dot{u}_{N}(t)\right\rangle_{V^{\prime}, V}, \quad \forall v \in V_{0}, \forall t \in\right] t_{n}, t_{n+1}\right] .
\end{aligned}
$$

In order to pass to the limit when $N$ tends to infinity, we note that this is equivalent to

$$
\int_{0}^{T}\left(a\left(\tilde{u}_{N}(t), v(t)\right)+j\left(\tilde{g}_{N}(t), v(t)\right)\right) d t \geq \int_{0}^{T}\left\langle\tilde{f}_{N}(t), v(t)\right\rangle_{V^{\prime}, V} d t, \quad \forall v \in L^{2}\left(0, T ; V_{0}\right),
$$

and for every $t \in[0, T]$,

$$
\int_{0}^{t}\left(a\left(\tilde{u}_{N}(s), \dot{u}_{N}(s)\right)+j\left(\tilde{g}_{N}(s), \dot{u}_{N}(s)\right)\right) d s=\int_{0}^{t}\left\langle\tilde{f}_{N}(s), \dot{u}_{N}(s)\right\rangle_{V^{\prime}, V} d s .
$$

Using the weak convergence (2.21) and (2.22) and the strong convergence in $L^{2}(0, T$; $\left.L^{2}\left(\Gamma_{3}\right)\right)$ of $\left(\tilde{g}_{N}\right)$ to $g$, the limit of assertion (2.25) can be easily obtained and we get

$$
\int_{0}^{T}(a(u(t), v(t))+j(g(t), v(t))) d t \geq \int_{0}^{T}\langle f(t), v(t)\rangle_{V^{\prime}, V} d t, \quad \forall v \in L^{2}\left(0, T ; V_{0}\right)
$$


In order to study the limit of (2.26), we denote by $N_{t}$ the integer with $t_{N_{t}}<t \leq t_{N_{t}+1}$ and we write on each subdivision $\left.] t_{n}, t_{n+1}\right], \tilde{u}_{N}(s)=u_{N}(s)+\left(t_{n+1}-s\right) \dot{u}_{N}(s)$. We then compute the integrals in (2.26) and we obtain

$$
\begin{aligned}
\frac{1}{2} a\left(u_{N}(t), u_{N}(t)\right) & -\frac{1}{2} a\left(u^{0}, u^{0}\right)+\frac{h^{2}}{2} \sum_{n=0}^{N_{t}-1} a\left(\frac{u_{n+1}-u_{n}}{h}, \frac{u_{n+1}-u_{n}}{h}\right) \\
& +\int_{0}^{t} j\left(\tilde{g}_{N}(s), \dot{u}_{N}(s)\right) d s \leq \int_{0}^{t}\left\langle\tilde{f}_{N}(s), \dot{u}_{N}(s)\right\rangle_{V^{\prime}, V} d s
\end{aligned}
$$

Using (2.22), we have for every $t \in[0, T]$,

$$
\liminf _{N} a\left(u_{N}(t), u_{N}(t)\right) \geq a(u(t), u(t))
$$

and since $\left(\dot{u}_{N}\right)_{N}$ is bounded in $L^{\infty}(0, T ; V)$, we have on one hand

$$
0 \leq h^{2} \sum_{n=0}^{N_{t}-1} a\left(\frac{u_{n+1}-u_{n}}{h}, \frac{u_{n+1}-u_{n}}{h}\right) \leq h^{2} \sum_{n=0}^{N-1} a\left(\frac{u_{n+1}-u_{n}}{h}, \frac{u_{n+1}-u_{n}}{h}\right)
$$

and on the other hand,

$$
h^{2} \sum_{n=0}^{N-1} a\left(\frac{u_{n+1}-u_{n}}{h}, \frac{u_{n+1}-u_{n}}{h}\right)=h \int_{0}^{T} a\left(\dot{u}_{N}(t), \dot{u}_{N}(t)\right) d t \rightarrow 0,
$$

thus

$$
\lim _{h \rightarrow 0} h^{2} \sum_{n=0}^{N_{t}-1} a\left(\frac{u_{n+1}-u_{n}}{h}, \frac{u_{n+1}-u_{n}}{h}\right)=0
$$

As $\left(\tilde{f}_{N}\right)$ strongly converge in $L^{2}\left(0, T ; V^{\prime}\right)$ to $f$, we get

$$
\int_{0}^{t}\left\langle\tilde{f}_{N}(s), \dot{u}_{N}(s)\right\rangle_{V^{\prime}, V} d s \longrightarrow \int_{0}^{t}\langle f(s), \dot{u}(s)\rangle_{V^{\prime}, V} d s .
$$

We now write

$$
\begin{aligned}
\int_{0}^{t} j\left(\tilde{g}_{N}(s), \dot{u}_{N}(s)\right) d s= & \int_{0}^{t}\left[j\left(\tilde{g}_{N}(s), \dot{u}_{N}(s)\right)-j\left(g(s), \dot{u}_{N}(s)\right)\right] d s \\
& +\int_{0}^{t} j\left(g(s), \dot{u}_{N}(s)\right) d s .
\end{aligned}
$$

On one hand, we have (since $\left(\tilde{g}_{N}\right)_{N}$ strongly converges to $g$ in $L^{2}\left(0, T ; L^{2}\left(\Gamma_{3}\right)\right)$ )

$$
\lim _{N} \int_{0}^{t}\left[j\left(\tilde{g}_{N}(s), \dot{u}_{N}(s)\right)-j\left(g(s), \dot{u}_{N}(s)\right)\right] d s=0,
$$


and on the other hand (since $j(g, \cdot)$ is convex)

$$
\liminf _{N} \int_{0}^{t} j\left(g(s), \dot{u}_{N}(s)\right) d s \geq \int_{0}^{t} j(g(s), \dot{u}(s)) d s .
$$

Passing to the inf-limit in (2.26), we obtain for every $t$ in $(0, T)$,

$$
\frac{1}{2} a(u(t), u(t))-\frac{1}{2} a\left(u^{0}, u^{0}\right)+\int_{0}^{t} j(g(s), \dot{u}(s)) d s \leq \int_{0}^{t}\langle f(s), \dot{u}(s)\rangle_{V^{\prime}, V} d s,
$$

and thus

$$
\int_{0}^{t} a(u(s), \dot{u}(s)) d s+\int_{0}^{t} j(g(s), \dot{u}(s)) d s \leq \int_{0}^{t}\langle f(s), \dot{u}(s)\rangle_{V^{\prime}, V} d s .
$$

Combining this inequality with (2.27), with $v=\dot{u} 1_{(0, t)}$ as test function, we prove that for almost every $t$,

$$
\int_{0}^{t} a(u(s), \dot{u}(s)) d s+\int_{0}^{t} j(g(s), \dot{u}(s)) d s=\int_{0}^{t}\langle f(s), \dot{u}(s)\rangle_{V^{\prime}, V} d s
$$

and hence for almost every $t$,

$$
a(u(t), \dot{u}(t))+j(g(t), \dot{u}(t))=\langle f(t), \dot{u}(t)\rangle_{V^{\prime}, V} .
$$

Assertions (2.27) and (2.40) yield to

$$
\begin{aligned}
a(u(t), v & -\dot{u}(t))+j(g(t), v)-j(g(t), \dot{u}(t)) \\
& \geq\langle f(t), v-\dot{u}(t)\rangle_{V^{\prime}, V}, \quad \forall v \in V_{0}, \text { a.e. } t \in(0, T),
\end{aligned}
$$

which proves that $u$ is solution of (2.6).

This ends the existence part of the proof of Proposition 2.1 in the case of regular data. If the data are $H^{1}$ with respect to the time variable, we can easily see that the proof can be adapted. The uniqueness of the solution can be obtained making the difference between two solutions and using Gronwall's lemma. This ends the proof of Proposition 2.2.

REMARK 2.4. Taking the inf-limit in (2.20), we have proved that for almost every $t \in] 0, T[$,

$$
\|\dot{u}(t)\|_{V} \leq c\left[\mu|\dot{g}(t)|_{L^{2}\left(\Gamma_{3}\right)}+\|\dot{f}(t)\|_{V^{\prime}}\right] .
$$

Proof of Proposition 2.3. We have to remark that in (2.19), the term

$$
\mu \int_{\Gamma_{3}}\left(g_{n}-g_{n+1}\right)\left|u_{n+1}-u_{n}\right| d a
$$

is negative under the assumptions made in Proposition 2.3. This leads to a uniform bound for the derivative with respect to the time variable. In order to obtain a similar bound for the solution $u$, it is then sufficient to take $v=0$ in (2.41). This ends the proof of Proposition 2.3. 
2.2. Existence and uniqueness in viscoplastic cases. In this section, we prove Proposition 2.1 applying a Banach fixed point theorem. For $\eta \in L^{2}(0, T$; $\mathscr{H})$, we consider the following auxiliary problem:

$$
\begin{aligned}
& \begin{aligned}
\sigma(t) & =\mathscr{E}\left(\varepsilon(u(t))-\varepsilon\left(u^{0}\right)\right)+\sigma^{0}+\int_{0}^{t} \eta(s) d s, \quad \text { a.e. } t \in(0, T) \\
(\sigma(t), & \varepsilon(v)-\varepsilon(\dot{u}(t)))_{\mathscr{H}}+j(g(t), v)-j(g(t), \dot{u}(t)) \\
& \geq\langle f(t), v-\dot{u}(t)\rangle_{V^{\prime}, V}, \quad \forall v \in V_{0}, \text { a.e. } t \in(0, T)
\end{aligned} \\
& u(0)=u^{0}
\end{aligned}
$$

Applying Proposition 2.2, we can easily see that there exists a unique solution $u \in$ $H^{1}\left(0, T ; V_{0}\right)$ to $(2.44)$. We introduce the map

$$
\begin{gathered}
\Lambda: L^{2}(0, T ; \mathscr{H}) \longrightarrow L^{2}(0, T ; \mathscr{H}) \\
\eta \longrightarrow G(\sigma, \varepsilon(u))
\end{gathered}
$$

where $(\sigma, u)=\left(\sigma_{\eta}, u_{\eta}\right)$ is the solution of system (2.44).

We prove the following lemma.

LEMmA 2.5. The map $\Lambda$ has a unique fixed point $\eta^{*} \in L^{2}(0, T ; \mathscr{H})$.

Proof. Let $\eta_{1}, \eta_{2} \in L^{2}(0, T ; \mathscr{H})$ and $t \in[0, T]$. For the sake of simplicity we denote $z_{i}(t)=\int_{0}^{t} \eta_{i}(s) d s, u_{i}=u_{\eta_{i}}, \sigma_{i}=\sigma_{\eta_{i}}$, for $i=1$,2. Using (2.44) and choosing as test function $v=\dot{u}_{i}$ in the inequality satisfied by $u_{j}$, we obtain

$$
\begin{aligned}
& a\left(u_{1}-u_{2}, \dot{u}_{1}-\dot{u}_{2}\right) \\
& \quad \leq-\frac{d}{d t}\left(z_{1}-z_{2}, \varepsilon\left(u_{1}\right)-\varepsilon\left(u_{2}\right)\right)_{\mathscr{H}}+\left(\eta_{1}-\eta_{2}, \varepsilon\left(u_{1}\right)-\varepsilon\left(u_{2}\right)\right)_{\mathscr{H}} .
\end{aligned}
$$

We deduce

$$
\begin{aligned}
c \mid u_{1}(t) & -\left.u_{2}(t)\right|_{V} ^{2} \\
& \leq\left|z_{1}(t)-z_{2}(t)\right|_{\mathscr{H}}+\int_{0}^{t}\left|\eta_{1}(s)-\eta_{2}(s)\right|{ }_{\mathscr{H}}\left|u_{1}(s)-u_{2}(s)\right|_{V} d s,
\end{aligned}
$$

for all $t \in[0, T]$. Hence,

$$
c\left|u_{1}(t)-u_{2}(t)\right|_{V}^{2} \leq \int_{0}^{t}\left|\eta_{1}(s)-\eta_{2}(s)\right|_{\mathscr{H}}^{2} d s+\int_{0}^{t}\left|u_{1}(s)-u_{2}(s)\right|_{V}^{2} d s,
$$

and, by Gronwall-type inequality, we find

$$
\left|u_{1}(t)-u_{2}(t)\right|_{V}^{2} \leq c \int_{0}^{t}\left|\eta_{1}(s)-\eta_{2}(s)\right|_{\mathscr{H}}^{2} d s .
$$

Using once more (2.44), we obtain

$$
\left|\sigma_{1}(t)-\sigma_{2}(t)\right|_{\mathscr{H}}^{2} \leq c \int_{0}^{t}\left|\eta_{1}(s)-\eta_{2}(s)\right|_{\mathscr{H}}^{2} d s .
$$


Therefore, using (1.8), (2.49), and (2.50), we get

$$
\left|\Lambda \eta_{1}(t)-\Lambda \eta_{2}(t)\right|_{\mathscr{H}}^{2} \leq c \int_{0}^{t}\left|\eta_{1}(s)-\eta_{2}(s)\right|_{\mathscr{H}}^{2} d s
$$

for all $t \in[0, T]$.

Iterating this inequality $n$ times we obtain

$$
\left|\Lambda^{n} \eta_{1}-\Lambda^{n} \eta_{2}\right|_{L^{2}(0, T ; \mathscr{H})}^{2} \leq \frac{c^{n} T^{n}}{n !}\left|\eta_{1}-\eta_{2}\right|_{L^{2}(0, T ; \mathscr{H})}^{2},
$$

which implies that for $n$ large enough a power $\Lambda^{n}$ of $\Lambda$ is a contraction in $L^{2}(0, T ; \mathscr{H})$ which proves Lemma 2.5 .

3. Proof of Theorem 1.1: the bilateral contact problem. In this section, we prove Theorem 1.1 beginning with the case where $f \in W^{1, \infty}\left(0, T ; V^{\prime}\right)$. We denote by $|\cdot|_{2}$ the $L^{2}\left(\Gamma_{3}\right)$-norm and we introduce the space

$$
\operatorname{Lip}=\left\{g \in C\left([0, T] ; L^{2}\left(\Gamma_{3}\right)\right)|\exists k>0, \forall(s, t) \in[0, T],| g(t)-\left.g(s)\right|_{2} \leq k|t-s|\right\}
$$

and for $k>0$,

$$
\operatorname{Lip}_{k}^{0}=\left\{g \in C\left([0, T] ; L^{2}\left(\Gamma_{3}\right)\right) \mid\left\{\begin{array}{l}
\forall(s, t) \in[0, T], \quad|g(t)-g(s)|_{2} \leq k|t-s|, \\
g(x, 0)=R\left(\sigma_{v}^{0}\right)(x) \text { on } \Gamma_{3} .
\end{array}\right\}\right.
$$

The set $\operatorname{Lip}_{k}^{0}$ is a closed convex subset of $C\left([0, T] ; L^{2}\left(\Gamma_{3}\right)\right)$ and $\operatorname{Lip}_{k}^{0} \subset W^{1, \infty}\left(0, T ; L^{2}\left(\Gamma_{3}\right)\right)$. We endow the space Lip with the norm $\sup _{0 \leq t \leq T}|g(t)|_{2}$.

Applying Proposition 2.1, for every $g \in$ Lip with $g(0, x)=R\left(\sigma_{v}^{0}\right)(x)$ on $\Gamma_{3}$, there exists a unique solution $(u, \sigma) \in W^{1, \infty}(0, T ; V) \times W^{1, \infty}(0, T ; \mathscr{H})$ to problem (2.44) which gives $R\left(\sigma_{v}\right) \in$ Lip. We then consider the map

$$
\begin{aligned}
T: \operatorname{Lip} & \longrightarrow \operatorname{Lip} \\
g & \longrightarrow R\left(\sigma_{v}\right) .
\end{aligned}
$$

It is clear that Theorem 1.1 will be proved (in regular case) if $T$ has a fixed point. We are now going to prove this with Schauder's theorem.

We begin with the following lemma.

LEMmA 3.1. Let $f \in W^{1, \infty}\left(0, T ; V^{\prime}\right)$. There exists $\mu_{0}>0$, such that for $0 \leq \mu \leq \mu_{0}$, there exists $k>0$, with

$$
T\left(\operatorname{Lip}_{k}^{0}\right) \subset \operatorname{Lip}_{k}^{0}
$$

Proof. We first recall that $(u, \sigma)$ is solution of (2.44) if for $0 \leq t \leq T$,

$$
\sigma(t)=\sigma^{0}-\mathscr{E} \varepsilon\left(u^{0}\right)+\mathscr{E} \varepsilon(u(t))+\int_{0}^{t} G(\sigma(s), \varepsilon(u(s))) d s,
$$


and for every $v \in V_{0}$,

$$
\begin{aligned}
& a(u(t), v-\dot{u}(t))+j(g(t), v)-j(g(t), \dot{u}(t)) \\
& \quad \geq\langle f(t), v-\dot{u}(t)\rangle_{V^{\prime}, V}-\left(\sigma^{0}-\mathscr{E} \varepsilon\left(u^{0}\right)+\int_{0}^{t} G(\sigma(s), \varepsilon(u(s))) d s, \varepsilon(v)-\varepsilon(\dot{u}(t))\right)_{\mathscr{L}} .
\end{aligned}
$$

First, we estimate solutions $\sigma$ and $u$. Input $v=0$ and then $v=2 \dot{u} \in V_{0}$ in (3.6), we get

$$
\begin{aligned}
& a(u(t), \dot{u}(t))+j(g(t), \dot{u}(t)) \\
& \quad=\langle f(t), \dot{u}(t)\rangle_{V^{\prime}, V}-\left(\sigma^{0}-\mathscr{E} \varepsilon\left(u^{0}\right)+\int_{0}^{t} G(\sigma(s), \varepsilon(u(s))) d s, \varepsilon(\dot{u}(t))\right)_{\mathscr{H}} .
\end{aligned}
$$

Integrating in time on $(0, t)$ for any $0<t<T$, we get

$$
\begin{aligned}
\frac{1}{2} a(u(t), u(t))+\int_{0}^{t} j(g(s), \dot{u}(s)) d s \\
=\langle f(t), u(t)\rangle_{V^{\prime}, V}-\int_{0}^{t}\langle\dot{f}(s), u(s)\rangle_{V^{\prime}, V} d s \\
\quad-\left\langle f(0), u^{0}\right\rangle_{V^{\prime}, V}+\frac{1}{2} a\left(u^{0}, u^{0}\right) \\
\quad-\left(\sigma^{0}-\mathscr{E} \varepsilon\left(u^{0}\right)+\int_{0}^{t} G(\sigma(s), \varepsilon(u(s))) d s, \varepsilon(u(t))\right)_{\mathscr{H}} \\
\quad+\left(\sigma^{0}-\mathscr{E} \varepsilon\left(u^{0}\right), \varepsilon\left(u^{0}\right)\right)_{\mathscr{H}} \\
\quad+\int_{0}^{t}(G(\sigma(s), \varepsilon(u(s))), \varepsilon(u(s)))_{\mathscr{H}} d s .
\end{aligned}
$$

Using (1.8) and writing $G(\sigma, \varepsilon(u))=[G(\sigma, \varepsilon(u))-G(0,0)]+G(0,0)$, there exists a constant $c>0$ which does not depend on $g$ and $k$ such that

$$
\begin{aligned}
\|u(t)\|_{V} \leq c[ & \left\|u^{0}\right\|_{V}+\left|\sigma^{0}\right|_{\mathscr{H}}+\|f\|_{W^{1, \infty}\left(0, T ; V^{\prime}\right)} \\
& \left.+|G(0,0)|_{\mathscr{H}}+\int_{0}^{t}\left(\|u(s)\|_{V}+|\sigma(s)|_{\mathscr{H}}\right) d s\right] .
\end{aligned}
$$

From (3.5), we obtain with $F(s)=\|u(s)\|_{V}+|\sigma(s)|_{\mathscr{H}_{1}}$ (since $\operatorname{Div} \sigma=\varphi_{1}$ )

$$
|\sigma(t)|_{\mathscr{H}_{1}} \leq c\left[\left\|u^{0}\right\|_{V}+\left|\sigma^{0}\right|_{\mathscr{H}_{1}}+|G(0,0)|_{\mathscr{H}}+\|f\|_{W^{1, \infty}\left(0, T ; V^{\prime}\right)}+\int_{0}^{t} F(s) d s\right] .
$$

Adding (3.9) and (3.10), we get

$$
F(t) \leq c\left[\left\|u^{0}\right\|_{V}+\|f\|_{W^{1, \infty}\left(0, T ; V^{\prime}\right)}+\left|\sigma^{0}\right|_{\mathscr{H}}+|G(0,0)|_{\mathscr{H}}+\int_{0}^{t} F(s) d s\right],
$$

which, with Gronwall's lemma, leads to

$$
\|u(s)\|_{V}+|\sigma(s)|_{\mathscr{H}_{1}} \leq c\left[\left\|u^{0}\right\|_{V}+\|f\|_{W^{1, \infty}\left(0, T ; V^{\prime}\right)}+\left|\sigma^{0}\right|_{\mathscr{H}}+|G(0,0)|_{\mathscr{H}}\right],
$$

where the constant $c>0$ does not depend on $k$ and the function $g$. 
Now, we estimate $\dot{\sigma}$ and $\dot{u}$. Using the constitutive law and hypothesis (1.8), we have on one hand (since Div $\dot{\sigma}=\dot{\varphi}_{1}$ )

$$
\begin{aligned}
|\dot{\sigma}(t)|_{\mathscr{H}_{1}} & \leq c\left[\|\dot{u}(t)\|_{V}+F(t)+|G(0,0)|_{\mathscr{H}}+|\dot{f}(t)|_{V^{\prime}}\right] \\
& \leq c\left[\|\dot{u}(t)\|_{V}+\left\|u^{0}\right\|_{V}+\|f\|_{W^{1, \infty}\left(0, T ; V^{\prime}\right)}+\left|\sigma^{0}\right|_{\mathscr{H}}+|G(0,0)|_{\mathscr{H}}\right] .
\end{aligned}
$$

On the other hand, (2.42) applied in the framework of the variational formulation (3.6) leads to

$$
\|\dot{u}(t)\|_{V} \leq c \mu|\dot{g}(t)|_{2}+c\left[|G(\sigma(t), \varepsilon(u(t)))|_{\mathscr{H}}+\left\|u^{0}\right\|_{V}+\left|\sigma^{0}\right|_{\mathscr{H}}+|\dot{f}(t)|_{V^{\prime}}\right] .
$$

Using the same argument as previously, there then exists a constant $c>0$ such that

$$
\left|R\left(\dot{\sigma}_{v}(t)\right)\right|_{2} \leq c|\dot{\sigma}(t)|_{\mathscr{H}_{1}} \leq c \mu k+c\left[\left\|u^{0}\right\|_{V}+\|f\|_{W^{1, \infty}\left(0, T ; V^{\prime}\right)}+\left|\sigma^{0}\right|_{\mathscr{H}}+|G(0,0)|_{\mathscr{H}}\right] .
$$

There exists $c, d>0$ which do not depend on $k$ and $\mu$ such that $T\left(\operatorname{Lip}_{k}^{0}\right) \subset \operatorname{Lip}_{c \mu k+d}^{0}$. In order to conclude, it is sufficient to prove that we can choose $k$ such that $c \mu k+d \leq k$. This is always possible if and only if

$$
0 \leq \mu<\frac{1}{c}
$$

which ends the proof of Lemma 3.1.

We prove the following lemma.

LEMMA 3.2. (i) For every $k>0$, there exists $C_{k}>0$ such that for every $\left(g_{1}, g_{2}\right) \in$ $\left(\operatorname{Lip}_{k}^{0}\right)^{2}$,

$$
\sup _{0 \leq t \leq T}\left(\left\|u_{1}(t)-u_{2}(t)\right\|_{V},\left|\sigma_{1}(t)-\sigma_{2}(t)\right|_{\mathscr{H}_{1}}\right) \leq C_{k} \sqrt{\left.\left\|u_{1}-u_{2}\right\|_{C\left([0, T] ; L^{2}\left(\Gamma_{3}\right) d\right.}\right)},
$$

where $u_{i}(i=1,2)$ is the displacement solution of (2.44) with $g_{i}$ as friction yield limit.

(ii) The application $T$ maps $\operatorname{Lip}_{k}^{0}$ in a compact set of $C\left([0, T] ; L^{2}\left(\Gamma_{3}\right)\right)$.

Proof. We first notice that $\operatorname{Lip}_{k}^{0}$ is bounded in $C\left([0, T] ; L^{2}\left(\Gamma_{3}\right)\right)$ and

$$
\sup _{0 \leq t \leq T}|g(t)|_{2} \leq\left|R\left(\sigma_{v}^{0}\right)\right|_{2}+k T
$$

We write $M=\left|R\left(\sigma_{v}^{0}\right)\right|_{2}+k T$.

(i) Using (3.5), (3.6), and (3.7), we can see that displacement $u_{i}$ for $i=1$ or $i=2$ satisfies the inequality

$$
\begin{aligned}
& a\left(u_{i}(t), v\right)+j\left(g_{i}(t), v\right) \\
& \quad \geq\langle f(t), v\rangle_{V^{\prime}, V}+\left(\sigma^{0}-\mathscr{E} \varepsilon\left(u^{0}\right)+\int_{0}^{t} G\left(\sigma_{i}(s), \varepsilon(u(s))\right) d s, \varepsilon(v)\right)_{\mathscr{H}} .
\end{aligned}
$$


We take $v=u_{2}-u_{1}$ in (3.19) for $i=1, v=u_{1}-u_{2}$ in the same inequality for $i=2$, and we add these two inequalities. We obtain

$$
\begin{aligned}
a\left(u_{2}(t)-\right. & \left.u_{1}(t), u_{2}(t)-u_{1}(t)\right) \\
\leq & j\left(g_{1}(t), u_{2}(t)-u_{1}(t)\right)+j\left(g_{2}(t), u_{2}(t)-u_{1}(t)\right) \\
& +\left(\int_{0}^{t}\left[G\left(\sigma_{2}(s), \varepsilon\left(u_{2}(s)\right)\right)-G\left(\sigma_{1}(s), \varepsilon\left(u_{1}(s)\right)\right)\right] d s, \varepsilon\left(u_{2}(t)\right)-\varepsilon\left(u_{1}(t)\right)\right)_{\mathscr{H}},
\end{aligned}
$$

and, with (1.8), we deduce

$$
\begin{aligned}
\left\|u_{2}(t)-u_{1}(t)\right\|_{V}^{2} \leq c & {\left[\mu M\left|u_{2}(t)-u_{1}(t)\right|_{L^{2}\left(\Gamma_{3}\right)^{d}}\right.} \\
& \left.+\int_{0}^{t}\left(\left\|u_{2}(s)-u_{1}(s)\right\|_{V}^{2}+\left|\sigma_{2}(s)-\sigma_{1}(s)\right|_{\mathscr{H}}^{2}\right) d s\right] \\
\leq c & {\left[\mu M\left\|u_{2}-u_{1}\right\|_{C\left([0, T] ; L^{2}\left(\Gamma_{3}\right)^{d}\right)}\right.} \\
& \left.+\int_{0}^{t}\left(\left\|u_{2}(s)-u_{1}(s)\right\|_{V}^{2}+\left|\sigma_{2}(s)-\sigma_{1}(s)\right|_{\mathscr{H}}^{2}\right) d s\right] .
\end{aligned}
$$

On the other hand,

$$
\begin{gathered}
\sigma_{2}(t)-\sigma_{1}(t)=\mathscr{E} \varepsilon\left(u_{2}-u_{1}\right)(t)+\int_{0}^{t}\left(G\left(\sigma_{2}(s), \varepsilon\left(u_{2}(s)\right)\right)-G\left(\sigma_{1}(s), \varepsilon\left(u_{1}(s)\right)\right)\right) d s, \\
\operatorname{Div}\left(\sigma_{2}(t)-\sigma_{1}(t)\right)=0,
\end{gathered}
$$

thus

$$
\begin{aligned}
\mid \sigma_{2}(t)- & \left.\sigma_{1}(t)\right|_{\mathscr{H}_{1}} \\
& \leq c\left[\left\|u_{2}(t)-u_{1}(t)\right\|_{V}+\int_{0}^{t}\left(\left\|u_{2}(s)-u_{1}(s)\right\|_{V}+\left|\sigma_{2}(s)-\sigma_{1}(s)\right|_{\mathscr{H}}\right) d s\right] .
\end{aligned}
$$

Adding (3.21) and (3.23) and using Gronwall's lemma, we deduce (3.17) with $C_{k}=$ $\mathcal{O}(k)+1$.

(ii) We proved in Lemma 3.1 (and in Proposition 2.1) that displacements and stresses $(u, \sigma)$ solution of (2.44) associated to friction bound $g \in \operatorname{Lip}_{k}^{0}$ are bounded in $W^{1, \infty}(0, T ; V) \times W^{1, \infty}\left(0, T ; \mathscr{H}_{1}\right)$. This proves that the set of the traces on $\Gamma_{3}$ of the displacements is relatively compact in $C\left([0, T] ; L^{2}\left(\Gamma_{3}\right)^{d}\right)$. Then let $\left(g_{n}\right)_{n}$ be a sequence of functions in $\operatorname{Lip}_{k}^{0}$. Denoting by $u_{n}$ and $\sigma_{n}$ the corresponding displacements and stresses, we can extract a subsequence of $\left(u_{n}\right)_{n}$ in order that their traces on $\Gamma_{3}$ strongly converge in $C\left([0, T] ; L^{2}\left(\Gamma_{3}\right)^{d}\right)$ and then realize a Cauchy sequence in that space. Using Lemma 3.2(i), we deduce that the corresponding subsequence of $\left(u_{n}\right)_{n}$ and $\left(\sigma_{n}\right)_{n}$ are Cauchy sequences in $C([0, T] ; V)$ and $C\left([0, T] ; \mathscr{H}_{1}\right)$, respectively, and then strongly converges in these spaces. Using the definition of the map $T$, this ends the proof of Lemma 3.2.

From now on, we choose $k>0$ and $\mu$ such that Lemma 3.1 is valid: we then have $T\left(\operatorname{Lip}_{k}^{0}\right) \subset \operatorname{Lip}_{k}^{0}$. Let $X_{k}$ be the closure in $C\left([0, T] ; L^{2}\left(\Gamma_{3}\right)\right)$ of $T\left(\operatorname{Lip}_{k}^{0}\right)$. We still have 
$T\left(X_{k}\right) \subset X_{k}$ and Lemma 3.2 proves that $X_{k}$ is compact in $C\left([0, T] ; L^{2}\left(\Gamma_{3}\right)\right)$. Applying Schauder fixed point theorem, Theorem 1.1 will be proved if $T$ is continuous on $X_{k}$. We prove that $T$ is continuous on $\operatorname{Lip}_{k}^{0}$. Let $g_{n}$ and $g$ be in $\operatorname{Lip}_{k}^{0}$ such that

$$
\lim _{n \rightarrow+\infty} \sup _{0 \leq t \leq T}\left|g_{n}(t)-g(t)\right|_{2}=0
$$

Using Lemma 3.2, the corresponding solution $u_{n}$ and $\sigma_{n}$ of (2.44) are bounded in $W^{1, \infty}(0, T ; V)$ and $W^{1, \infty}\left(0, T ; \mathscr{H}_{1}\right)$, respectively, and are relatively compact in $C([0, T]$; $V)$ and $C\left([0, T] ; \mathscr{H}_{1}\right)$, respectively. We can extract a subsequence still denoted by $\left(u_{n}, \sigma_{n}\right)$ which converges weakly -* in $W^{1, \infty}(0, T ; V) \times W^{1, \infty}\left(0, T ; \mathscr{H}_{1}\right)$ and strongly in $C([0, T] ; V) \times C\left([0, T] ; \mathscr{H}_{1}\right)$ to a point $(u, \sigma) \in W^{1, \infty}(0, T, V) \times W^{1, \infty}\left(0, T ; \mathscr{H}_{1}\right)$.

We still have to prove that $(u, \sigma)$ is solution of (2.44) associated to $g$. Using (1.8), we can easily prove that

$$
\lim _{n \rightarrow+\infty} \sup _{0 \leq t \leq T}\left|G\left(\sigma_{n}, \varepsilon\left(n_{u}\right)\right)-G(\sigma, \varepsilon(u))\right|_{\mathscr{H}}=0
$$

and thus

$$
\dot{\sigma}=\mathscr{E} \varepsilon(\dot{u})+G(\sigma, \varepsilon(u)) .
$$

In order to pass to the inf-limit in inequality (2.44), we write it for $v \in L^{2}\left(0, T ; V_{0}\right)$ and integrate in time on the interval $(0, T)$. The only delicate point is the term

$$
\int_{0}^{T} j\left(g_{n}(t), \dot{u}_{n}(t)\right) d t .
$$

We write

$$
\begin{aligned}
\int_{0}^{T} j\left(g_{n}(t), \dot{u}_{n}(t)\right) d t= & \int_{0}^{T}\left[j\left(g_{n}(t), \dot{u}_{n}(t)\right)-j\left(g(t), \dot{u}_{n}(t)\right)\right] d t \\
& +\int_{0}^{T} j\left(g(t), \dot{u}_{n}(t)\right) d t
\end{aligned}
$$

Using (3.24), the boundedness of $\left(\dot{u}_{n}\right)_{n}$ in $L^{2}(0, T ; V)$ and the convexity of $j$ we get

$$
\liminf _{n \rightarrow+\infty} \int_{0}^{T} j\left(g_{n}(t), \dot{u}_{n}(t)\right) d t \geq \int_{0}^{T} j(g(t), \dot{u}(t)) d t
$$

We can see that (as in Section 2), this is sufficient to prove that $(u, \sigma)$ is a solution of (2.44) and thus $T(g)$ is a strong limit point of $\left(T\left(g_{n}\right)\right)_{n}$. Since (2.44) possesses a unique solution and that the sequence $\left(T\left(g_{n}\right)\right)_{n}$ is relatively compact, we conclude that the whole sequence converges and

$$
\lim _{n \rightarrow+\infty} T\left(g_{n}\right)=T(g) \text { in } C\left([0, T] ; L^{2}\left(\Gamma_{3}\right)\right) .
$$

The map $T$ is continuous on the compact set $X_{k}$ of $C\left([0, T] ; L^{2}\left(\Gamma_{3}\right)\right)$, it then admits a fixed point which solves Theorem 1.1 in regular cases. 
The case of data $f \in H^{1}\left(0, T ; V^{\prime}\right)$ can easily be obtained by a density argument (approximating $f$ by a sequence $\left(f_{n}\right)_{n}$ of functions in $W^{1, \infty}\left(0, T ; V^{\prime}\right)$ ).

4. Proof of Theorem 1.2: Signorini's contact model. In this section, we are concerned with problem (1.5) and variational formulation (1.19). The steps of proofs used in the previous section are no more valid and the reason is that, even if we could prove, without a main change, existence of a solution to (2.3) where the bilateral contact condition $u_{v}=0$ on $\Gamma_{3}$ would be replaced by the Signorini's model $u_{v} \leq 0, \sigma_{v} \leq 0$, and $u_{v} \sigma_{v}=0$ on $\Gamma_{3}$, we could not prove uniqueness of a solution. Therefore, we could only define a multivalued map $T: \mathfrak{g} \rightarrow\left\{R\left(\sigma_{v}\right)\right\}$. As we could not prove the convexity of each image set, we could not use Kakutani's fixed point theorem.

Another fact which deserves to be point out concerns variational formulations for unilateral contact problems. This can be found in [4] which we will follow: the proof of Theorem 1.2 consists first in a time discretization method which allows to construct a (convenient) sequence followed secondly by an asymptotic analysis. As we will see, the study of this last step needs the use of two equivalent variational formulations of the same problem.

We first describe the iterative method. Recall that $T>0$ is fixed, $N \in \mathbb{N}$ is an integer and $h=T / N$. We construct a sequence $\left(u_{n}, \sigma_{n}\right)_{0 \leq n \leq N}$ of elements $u_{n} \in V$ and $\sigma_{n} \in \mathscr{H}_{1}$ with the following process: $u_{0}=u^{0}$ and $\sigma_{0}=\sigma^{0}$ are the given initial data which appear in (1.5).

Construction of $\left(u_{1}, \sigma_{1}\right)$. We introduce the continuous linear form on $V$ defined by

$$
b_{1}(w)=\left(\sigma_{0}-\mathscr{E} \varepsilon\left(u_{0}\right)+h G\left(\sigma_{0}, \varepsilon\left(u_{0}\right)\right), \varepsilon(w)\right)_{\mathscr{H}}
$$

and we consider the variational formulation

$$
\begin{aligned}
& \sigma_{1}=\mathscr{E} \varepsilon\left(u_{1}\right)+\sigma_{0}-\mathscr{E} \varepsilon\left(u_{0}\right)+h G\left(\sigma_{0}, \varepsilon\left(u_{0}\right)\right), \\
& \begin{aligned}
a\left(u_{1}, w-u_{1}\right)+j\left(R\left(\sigma_{1 v}\right), w-u_{0}\right)-j\left(R\left(\sigma_{1 v}\right), u_{1}-u_{0}\right)+b_{1}\left(w-u_{1}\right) \\
\quad \geq\left\langle f_{1}, w-u_{1}\right\rangle_{V^{\prime}, V}+\left\langle\sigma_{1 v}, w_{v}-u_{1 v}\right\rangle, \quad \forall w \in V,
\end{aligned} \\
& \left\langle\sigma_{1 v}, w_{v}-u_{1 v}\right\rangle_{V^{\prime}, V}, \geq 0, \quad \forall w \in K .
\end{aligned}
$$

We prove the following proposition.

Proposition 4.1. There exists $\mu_{0}>0$ such that for every $u^{0} \in K, \sigma^{0} \in \mathscr{H}_{1}$, every $f_{1} \in V^{\prime}$, and for $0<\mu \leq \mu_{0}$, problem (4.2) has a unique solution $\left(u_{1}, \sigma_{1}\right) \in K \times \mathscr{H}_{1}$.

REMARK 4.2. The boundary $\mu_{0}$ depends only on $\mathscr{E}, R$ and $\Omega$.

Proof of Proposition 4.1. For $g \in L^{2}\left(\Gamma_{3}\right)$, we consider the auxiliary problem

$$
\begin{gathered}
a\left(u_{1}, w-u_{1}\right)+j\left(g, w-u_{0}\right)-j\left(g, u_{1}-u_{0}\right)+b_{1}\left(w-u_{1}\right) \\
\geq\left\langle f_{1}, w-u_{1}\right\rangle_{V^{\prime}, V}+\left\langle\sigma_{1 v}, w_{v}-u_{1 v}\right\rangle, \quad \forall w \in V, \\
\left\langle\sigma_{1 v}, w_{v}-u_{1 v}\right\rangle \geq 0, \quad \forall w \in K .
\end{gathered}
$$


Since for $w \in K,\left\langle\sigma_{1 v}, w_{v}-u_{1 v}\right\rangle \geq 0$, a solution of (4.3) is necessarily solution of

$$
\begin{aligned}
a\left(u_{1}, w-u_{1}\right)+j\left(g, w-u_{0}\right)-j\left(g, u_{1}-u_{0}\right)+b_{1}\left(w-u_{1}\right) & \\
& \geq\left\langle f_{1}, w-u_{1}\right\rangle_{V^{\prime}, V}, \quad \forall w \in K .
\end{aligned}
$$

It is classical that (4.4) has a unique solution which is the minimizer over $K$ of the (strictly convex coercive and continuous) functional

$$
J(w)=\frac{1}{2} a(w, w)+j\left(g, w-u_{0}\right)+b_{1}(w)-\left\langle f_{1}, w\right\rangle_{V^{\prime}, V}
$$

Writing $\sigma_{1}=\mathscr{E} \varepsilon\left(u_{1}\right)+\sigma_{0}-\mathscr{E} \varepsilon\left(u_{0}\right)+h G\left(\sigma_{0}, \varepsilon\left(u_{0}\right)\right)$, inequality (4.4) can be written as

$$
\left(\sigma_{1}, \varepsilon(w)-\varepsilon\left(u_{1}\right)\right)_{\mathscr{H}}+j\left(g, w-u_{0}\right)-j\left(g, u_{1}-u_{0}\right) \geq\left\langle f_{1}, w-u_{1}\right\rangle_{V^{\prime}, V}, \quad \forall \omega \in K .
$$

Equivalence between (4.3) and (4.4) can be seen using the imbedding $\mathscr{D}(\Omega)^{d} \subset K$ and the density of the set $\left\{\left.u_{\tau}\right|_{\Gamma_{3}}, u \in K\right\}$ in $L^{2}\left(\Gamma_{3}\right)^{d}$ (see [4]).

We then consider the map

$$
\begin{aligned}
T_{1}: L^{2}\left(\Gamma_{3}\right) & \longrightarrow L^{2}\left(\Gamma_{3}\right) \\
g & \longrightarrow R\left(\sigma_{1 v}\right),
\end{aligned}
$$

where $\sigma_{1}$ is defined above. We prove that for $\mu$ small enough, the map $T_{1}$ has a unique fixed point. We use the Banach fixed point theorem. It is clearly sufficient to prove that for $\mu$ small enough,

$$
\left\|u_{1}-u_{1}^{\prime}\right\|_{V} \leq k\left|g-g^{\prime}\right|_{2}
$$

with $0<k<1$ and where $u_{1}$ (and $u_{1}^{\prime}$ ) are the solution of (4.3), with respect to $g$ (and $\left.g^{\prime}\right)$, respectively.

Let $w=u_{1}^{\prime} \in K$ in the inequality satisfied by $u_{1}$ and $w=u_{1} \in K$ in the one satisfied by $u_{1}^{\prime}$, add them to obtain

$$
a\left(u_{1}-u_{1}^{\prime}, u_{1}-u_{1}^{\prime}\right) \leq \mu \int_{\Gamma_{3}}\left|g-g^{\prime}\right|\left|u_{1, \tau}-u_{1, \tau}^{\prime}\right| d a .
$$

This last inequality proves that there exists $\mu_{0} \geq 0$ independent on $u^{0}, \sigma^{0}$, and $f_{1}$ such that for $0<\mu \leq \mu_{0}, T_{1}$ is contractive. This ends the proof of Proposition 4.1.

Construction of $\left(u_{n+1}, \sigma_{n+1}\right)$. We suppose that we have construct in a unique way $\left(u_{1}, \sigma_{1}\right) \cdots\left(u_{n}, \sigma_{n}\right)$ in $K \times \mathscr{H}$ solutions of the following systems for $1 \leq k \leq n$ :

$$
\begin{gathered}
\sigma_{k}=\mathscr{E} \varepsilon\left(u_{k}\right)+\sigma_{0}-\mathscr{E} \varepsilon\left(u_{0}\right)+h\left(G\left(\sigma_{0}, \varepsilon\left(u_{0}\right)\right)+\cdots+G\left(\sigma_{k-1}, \varepsilon\left(u_{k-1}\right)\right)\right), \\
\begin{aligned}
a\left(u_{k}, w-u_{k}\right)+j\left(R\left(\sigma_{k v}\right), w-u_{k-1}\right)-j\left(R\left(\sigma_{k v}\right), u_{k}-u_{k-1}\right)+b_{k}\left(w-u_{k}\right) \\
\quad \geq\left\langle f_{k}, w-u_{k}\right\rangle_{V^{\prime}, V}+\left\langle\sigma_{k v}, w_{v}-u_{k v}\right\rangle, \quad \forall w \in V,
\end{aligned} \\
\left\langle\sigma_{k v}, w_{v}-u_{k v}\right\rangle \geq 0, \quad \forall w \in K,
\end{gathered}
$$


where

$$
b_{k}(w)=\left(\sigma_{0}-\mathscr{E} \varepsilon\left(u_{0}\right)+h\left(G\left(\sigma_{0}, \varepsilon\left(u_{0}\right)\right)+\cdots+G\left(\sigma_{k-1}\right), \varepsilon\left(u_{k-1}\right)\right), \varepsilon(w)\right)_{\mathscr{H}} .
$$

For $g \in L^{2}\left(\Gamma_{3}\right)$ and $b_{n+1}(w)=b_{n}(w)+h\left(G\left(\sigma_{n}, \varepsilon\left(u_{n}\right)\right), \varepsilon(w)\right)_{\mathscr{H}}$, we then consider the following problem at step $n+1$ :

$$
\begin{gathered}
\sigma_{n+1}=\mathscr{E} \varepsilon\left(u_{n+1}\right)+\sigma_{0}-\mathscr{E} \varepsilon\left(u_{0}\right)+h\left(G\left(\sigma_{0}-\varepsilon\left(u_{0}\right)\right)+\cdots+G\left(\sigma_{n}, \varepsilon\left(u_{n}\right)\right)\right) \\
a\left(u_{n+1}, w-u_{n+1}\right)+j\left(g, w-u_{n}\right)-j\left(g, u_{n+1}-u_{n}\right)+b_{n+1}\left(w-u_{n+1}\right) \\
\geq\left\langle f_{n+1}, w-u_{n+1}\right\rangle_{V^{\prime}, V}+\left\langle\sigma_{n+1 v}, w_{v}-u_{n+1 v}\right\rangle, \quad \forall w \in V, \\
\left\langle\sigma_{n+1 v}, w_{v}-u_{n+1 v}\right\rangle \geq 0, \quad \forall w \in K .
\end{gathered}
$$

For each $g \in L^{2}\left(\Gamma_{3}\right)$, we prove (as in the first step) that (4.12) has a unique solution $u_{n+1} \in K$ minimizing over $K$ the functional

$$
J(w)=\frac{1}{2} a(w, w)+j\left(g, w-u_{n}\right)+b_{n+1}(w)-\left\langle f_{n+1}, w\right\rangle_{V^{\prime}, V} .
$$

We then define $\sigma_{n+1}=\mathscr{E} \varepsilon\left(u_{n+1}\right)+\sigma_{0}-\mathscr{E} \varepsilon\left(u_{0}\right)+h\left(G\left(\sigma_{0}, \varepsilon\left(u_{0}\right)\right)+\cdots+G\left(\sigma_{n}, \varepsilon\left(u_{n}\right)\right)\right)$ and we consider the map

$$
\begin{aligned}
T_{n+1}: L^{2}\left(\Gamma_{3}\right) & \longrightarrow L^{2}\left(\Gamma_{3}\right) \\
g & \longrightarrow R\left(\sigma_{n+1 v}\right) .
\end{aligned}
$$

Following the first step, we can prove that $T_{n+1}$ is contractive for $\mu \leq \mu_{0}$ (independent on $n$ and $\mu_{0}$ is the same as in the first step) and therefore possesses a unique fixed point. We denote by $\left(\sigma_{n+1}, u_{n+1}\right)$ the corresponding solution, it solves (in a unique way)

$$
\begin{aligned}
& \sigma_{n+1}=\mathscr{E} \varepsilon\left(u_{n+1}\right)+\sigma_{0}-\mathscr{E} \varepsilon\left(u_{0}\right)+h\left(G\left(\sigma_{0}, \varepsilon\left(u_{0}\right)\right)+\cdots+G\left(\sigma_{n}, \varepsilon\left(u_{n}\right)\right)\right), \\
& \begin{aligned}
a\left(u_{n+1}, w-u_{n+1}\right)+j\left(R \sigma_{n+1 v}, w-u_{n}\right)-j\left(R \sigma_{n+1 v}, u_{n+1}-u_{n}\right)+b_{n+1}\left(w-u_{n+1}\right) \\
\geq\left\langle f_{n+1}, w-u_{n+1}\right\rangle_{V^{\prime}, V}+\left\langle\sigma_{n+1 v}, w_{v}-u_{n+1 v}\right\rangle, \quad \forall w \in V
\end{aligned} \\
& \left\langle\sigma_{n+1 v}, w_{v}-u_{n+1 v}\right\rangle \geq 0, \quad \forall w \in K .
\end{aligned}
$$

With this iterative process, we construct a sequence $\left(u_{n}, \sigma_{n}\right)_{0 \leq n \leq N}$ in $K^{N+1} \times \mathscr{H}_{1}^{N+1}$ in a unique way.

In what follows, for a given sequence $\left(\xi_{n}\right)_{0 \leq n \leq N}$ of elements of a Banach $X$, we denote by $\xi_{N}, \tilde{\xi}_{N}$, and $\bar{\xi}_{N}$ the following functions defined on $[0, T]$ by

$$
\begin{aligned}
& \xi_{N}(t, x)=\xi_{n}+\frac{t-t_{n}}{h}\left(\xi_{n+1}-\xi_{n}\right), \\
& \tilde{\xi}_{N}(t, x)=\xi_{n+1}, \\
& \bar{\xi}_{N}(t, x)=\xi_{n},
\end{aligned}
$$

for $t_{n}<t \leq t_{n+1}$ and $0 \leq n \leq N-1$. The function (and only this one) $\xi_{N}$ is in $H^{1}(0, T ; X)$. 
This is done for $\left(u_{n}\right)_{n},\left(\sigma_{n}\right)_{n}$, and $\left(f_{n}\right)_{n}$ and we recall that forces $f_{N}$ strongly converge to $f$ in $H^{1}\left(0, T ; V^{\prime}\right)$ whereas $\tilde{f}_{N}$ and $\bar{f}_{N}$ only converge to $f$ in $L^{2}\left(0, T ; V^{\prime}\right)$.

We prove the following lemma.

LEMMA 4.3. The sequences $\left(u_{N}\right)_{N}$ and $\left(\sigma_{N}\right)_{N}$ are bounded, respectively in $H^{1}(0, T ; V)$ and $H^{1}\left(0, T ; \mathscr{H}_{1}\right)$ and any weak limit point $(u, \sigma)$ of these sequences is solution of problem (1.5).

Proof. We first notice that, with $w=0$ and then $w=2 u_{n+1} \in K$ in (4.15c), we obtain for $1 \leq n \leq N$

$$
\left\langle\sigma_{n v}, u_{n v}\right\rangle=0
$$

We now input $w=u_{n} \in K$ in inequality (4.15) satisfied by $u_{n+1}$ and $w=u_{n+1} \in K$ in inequality (4.10) satisfied by $u_{n}$ if $n \geq 1$ or in (1.20) if $n=0$, we add them and we get the following: if $n \geq 1$,

$$
\begin{aligned}
& a\left(u_{n+1}-u_{n}, u_{n+1}-u_{n}\right) \\
& \leq j\left(R \sigma_{n v}, u_{n+1}-u_{n-1}\right)-j\left(R \sigma_{n v}, u_{n}-u_{n-1}\right)-j\left(R \sigma_{n+1 v}, u_{n+1}-u_{n}\right) \\
&+h\left(G\left(\sigma_{n}, \varepsilon\left(u_{n}\right)\right), \varepsilon\left(u_{n+1}\right)-\varepsilon\left(u_{n}\right)\right)_{\mathscr{H}}+\left\langle f_{n+1}-f_{n}, u_{n+1}-u_{n}\right\rangle_{V^{\prime}, V}, \\
& a\left(u_{1}-u_{0}, u_{1}-u_{0}\right) \\
& \leq j\left(R \sigma_{0 v}, u_{1}-u_{0}\right)-j\left(R \sigma_{1 v}, u_{1}-u_{0}\right) \\
&+\left\langle f_{1}-f_{0}, u_{1}-u_{0}\right\rangle_{V^{\prime}, V}+h\left(G\left(\sigma^{0}, \varepsilon\left(u^{0}\right)\right), \varepsilon\left(u_{1}\right)-\varepsilon\left(u_{0}\right)\right)_{\mathscr{H}} .
\end{aligned}
$$

Hence

$$
\begin{aligned}
\left\|\frac{u_{n+1}-u_{n}}{h}\right\|_{V}^{2} \leq & \mu \int_{\Gamma_{3}}\left|\frac{R \sigma_{n v}-R \sigma_{(n+1) v}}{h}\right|\left|\frac{u_{(n+1)_{\tau}}-u_{n \tau}}{h}\right| d a \\
& +\left(G\left(\sigma_{n}, \varepsilon\left(u_{n}\right)\right), \frac{\varepsilon\left(u_{n+1}-u_{n}\right)}{h}\right)_{\mathscr{H}} \\
& +\left\|\frac{f_{n+1}-f_{n}}{h}\right\|_{V^{\prime}}\left\|\frac{u_{n+1}-u_{n}}{h}\right\|_{V} .
\end{aligned}
$$

So, we have

$$
\begin{aligned}
\left\|\frac{u_{n+1}-u_{n}}{h}\right\|_{V} \leq & c_{0} \mu\left\|\frac{\sigma_{n}-\sigma_{n+1}}{h}\right\|_{\mathscr{H}_{1}}+\|G(0,0)\|_{\mathscr{H}} \\
& +\left\|\frac{f_{n+1}-f_{n}}{h}\right\|_{V^{\prime}}+L\left[\left\|\sigma_{n}\right\|_{\mathscr{H}}+\left\|u_{n}\right\|_{V}\right] .
\end{aligned}
$$

Since for $t_{n}<t \leq t_{n+1}$, we have $\bar{u}_{N}(t)=u_{n}=u_{N}\left(t_{n}\right)=u_{N}(0)+\int_{0}^{t_{n}} \dot{u}_{N}(s) d s$, we then get

$$
\begin{aligned}
\left\|\dot{u}_{N}(t)\right\|_{V} \leq & c_{0} \mu\left\|\dot{\sigma}_{N}(t)\right\|_{\mathscr{H}_{1}}+\|G(0,0)\|\left\|_{\mathscr{H}}+\right\| \dot{f}_{N}(t) \|_{V^{\prime}} \\
& +\left\|\sigma^{0}\right\|_{\mathscr{H}}+\left\|u^{0}\right\|_{V}+\int_{0}^{t}\left[\left\|\dot{u}_{N}(s)\right\|_{V}+\left\|\dot{\sigma}_{N}(s)\right\|_{\mathscr{H}}\right] d s .
\end{aligned}
$$

On the other hand, a simple computation gives

$$
\dot{\sigma}_{N}(t)=\mathscr{E} \varepsilon\left(\dot{u}_{N}(t)\right)+G\left(\bar{\sigma}_{N}(t), \varepsilon\left(\bar{u}_{N}(t)\right)\right),
$$


from which we deduce that

$$
\begin{aligned}
\left\|\dot{\sigma}_{N}(t)\right\|_{\mathscr{H}} \leq c_{0}( & \left\|\dot{u}_{N}(t)\right\|_{V}+\left\|\sigma^{0}\right\|_{\mathscr{H}}+\left\|u^{0}\right\|_{V} \\
& \left.+\|G(0,0)\|_{\mathscr{H}}+\int_{0}^{t}\left[\left\|\dot{u}_{N}(s)\right\|_{V}+\left\|\dot{\sigma}_{N}(s)\right\|_{\mathscr{H}}\right] d s\right) .
\end{aligned}
$$

Combining these two last inequalities, we obtain the existence of a constant $c_{0}>0$ such that for every $0<\mu$, we have with

$$
\begin{aligned}
& F_{N}(t)=\left[\int_{0}^{t}\left(\left\|\dot{\sigma}_{N}(s)\right\|_{\mathscr{H}}^{2}+\left\|\dot{u}_{N}(s)\right\|_{V}^{2}\right) d s\right]^{1 / 2}, \\
& F_{N}(t) \leq c_{0}\left(\mu F_{N}(t)+\left\|f_{N}\right\|_{H^{1}\left(0, T ; V^{\prime}\right)}+\left\|\sigma^{0}\right\|_{\mathscr{H}_{1}}+\left\|u^{0}\right\|_{V}+\|G(0,0)\|_{\mathscr{H}}+\int_{0}^{t} F_{N}(s) d s\right) .
\end{aligned}
$$

Using Gronwall's lemma and for $\mu$ small enough, we deduce that for $f \in H^{1}\left(0, T ; V^{\prime}\right)$, the sequence $\left(F_{N}\right)_{N}$ is bounded in $L^{2}(0, T)$ and for $f \in W^{1, \infty}\left(0, T ; V^{\prime}\right)$, the sequence $\left(F_{N}\right)_{N}$ is bounded in $L^{\infty}(0, T)$. Since $u^{0}$ and $\sigma^{0}$ are fixed, we deduce the boundedness of $u_{N}$ and $\sigma_{N}$, respectively in $H^{1}(0, T ; V)$ and $H^{1}\left(0, T ; \mathscr{H}_{1}\right)$ if $f$ is in $H^{1}\left(0, T ; V^{\prime}\right)$ (and respectively in $W^{1, \infty}(0, T ; V)$ and $W^{1, \infty}\left(0, T ; \mathscr{H}_{1}\right)$ if the force $f$ is in $\left.W^{1, \infty}\left(0, T ; V^{\prime}\right)\right)$. The proof of Lemma 4.3 needs the following lemma.

LEMMA 4.4. Any weak limit point in $H^{1}(0, T ; V) \times H^{1}\left(0, T ; \mathscr{H}_{1}\right)$ of the sequence $\left(u_{N}, \sigma_{N}\right)_{N}$ is a strong limit point in $L^{2}([0, T] ; V) \times L^{2}\left([0, T] ; \mathscr{H}_{1}\right)$.

Proof. Denote by $(u, \sigma)$ a weak limit point of the sequence $\left(u_{N}, \sigma_{N}\right)_{N}$ in $H^{1}(0, T ; V) \times H^{1}\left(0, T ; \mathscr{H}_{1}\right)$. We first prove some estimates that will be useful. For simplicity, we denote by $z_{N} \in L^{2}(0, T ; \mathscr{H})$ the tensor

$$
z_{N}(t)=\sigma^{0}-\mathscr{E} \varepsilon\left(u^{0}\right)+\int_{0}^{t} G\left(\bar{\sigma}_{N}(s), \varepsilon\left(\bar{u}_{N}\right)(s)\right) d s .
$$

Multiplying (4.15) by $1 / h$ and rescaling with $v=\left(w-u_{n}\right) / h$, we can see that $u_{N}$ and $\sigma_{N}$ are solutions of

$$
\begin{aligned}
& \dot{\sigma}_{N}=\mathscr{E} \varepsilon\left(\dot{u}_{N}\right)+G\left(\bar{\sigma}_{N}, \varepsilon\left(\bar{u}_{N}\right)\right), \\
& \begin{aligned}
a\left(\tilde{u}_{N}(t), v-\dot{u}_{N}(t)\right)+ & j\left(R \tilde{\sigma}_{N}(t), v\right)-j\left(R \tilde{\sigma}_{N}(t), \dot{u}_{N}(t)\right)+\left(z_{N}(t), \varepsilon(v)-\varepsilon\left(\dot{u}_{N}(t)\right)\right)_{\mathscr{H}} \\
& \geq\left\langle\tilde{f}_{N}(t), v-\dot{u}_{N}(t)\right\rangle_{V^{\prime}, V}+\left\langle\tilde{\sigma}_{N v}, v_{v}-\dot{u}_{N v}\right\rangle, \quad \forall v \in V
\end{aligned} \\
& \left\langle\tilde{\sigma}_{N v}(t), w_{v}-\tilde{u}_{N v}(t)\right\rangle \geq 0, \quad \forall w \in K .
\end{aligned}
$$

Since for $t_{n}<t \leq t_{n+1}$, we have

$$
\left\|u_{N}(t)-\tilde{u}_{N}(t)\right\|_{V} \leq \frac{T}{N}\left\|\dot{u}_{N}(t)\right\|_{V}
$$

with $\left(\dot{u}_{N}\right)_{N}$ bounded in $L^{2}(0, T ; V)$, Lemma 4.4 will be proved if we prove that $(u, \sigma)$ is a strong limit point of $\left(\tilde{u}_{N}, \tilde{\sigma}_{N}\right)_{N}$ in $L^{2}([0, T] ; V) \times L^{2}\left([0, T] ; \mathscr{H}_{1}\right)$. 
If we input $v=0$ and then $v=2 \dot{u}_{N}$ in (4.26), we obtain that for all $v \in V$

$$
a\left(\tilde{u}_{N}(t), v\right)+j\left(R \tilde{\sigma}_{N v}(t), v\right)+\left(z_{N}(t), \varepsilon(v)\right)_{\mathscr{H}} \geq\left\langle\tilde{f}_{N}(t), v\right\rangle_{V^{\prime}, V}+\left\langle\tilde{\sigma}_{N v}(t), v_{v}\right\rangle \text {. }
$$

Let $P>N$ be an integer. We first consider $v=\tilde{u}_{P}(t)-\tilde{u}_{N}(t)$ in inequality (4.28) satisfied by $\tilde{u}_{N}$. Since $\tilde{u}_{P}(t) \in K$, we have $\left\langle\tilde{\sigma}_{N v}, \tilde{u}_{P v}-\tilde{u}_{N v}\right\rangle \geq 0$ and we get

$$
\begin{gathered}
a\left(\tilde{u}_{N}(t), \tilde{u}_{P}(t)-\tilde{u}_{N}(t)\right)+j\left(R \tilde{\sigma}_{N v}(t), \tilde{u}_{P}(t)-\tilde{u}_{N}(t)\right)+\left(z_{N}(t), \varepsilon\left(\tilde{u}_{P}\right)(t)-\varepsilon\left(\tilde{u}_{N}\right)(t)\right)_{\mathscr{H}} \\
\geq\left\langle\tilde{f}_{N}(t), \tilde{u}_{P}(t)-\tilde{u}_{N}(t)\right\rangle_{V^{\prime}, V} .
\end{gathered}
$$

We now consider inequality (4.28) satisfied by $u_{P}$ in which we take $v=\tilde{u}_{N}(t)-\tilde{u}_{P}(t)$ and we obtain

$$
\begin{gathered}
a\left(\tilde{u}_{P}(t), \tilde{u}_{N}(t)-\tilde{u}_{P}(t)\right)+j\left(R \tilde{\sigma}_{P V}(t), \tilde{u}_{N}(t)-\tilde{u}_{P}(t)\right)+\left(z_{P}(t), \varepsilon\left(\tilde{u}_{N}(t)\right)-\varepsilon\left(\tilde{u}_{P}\right)(t)\right)_{\mathscr{H}} \\
\geq\left\langle\tilde{f}_{P}(t), \tilde{u}_{N}(t)-\tilde{u}_{P}(t)\right\rangle_{V^{\prime}, V} .
\end{gathered}
$$

Adding (4.29) and (4.30), we get

$$
\begin{aligned}
a\left(\tilde{u}_{N}-\tilde{u}_{P}, \tilde{u}_{N}-\tilde{u}_{P}\right) \leq & \left\langle\tilde{f}_{N}-\tilde{f}_{P}, \tilde{u}_{N}-\tilde{u}_{P}\right\rangle_{V^{\prime}, V}-\left(z_{N}-z_{P}, \varepsilon\left(\tilde{u}_{N}\right)-\varepsilon\left(\tilde{u}_{P}\right)\right)_{\mathscr{H}} \\
& +j\left(R \tilde{\sigma}_{P, v}, \tilde{u}_{N}-\tilde{u}_{P}\right)+j\left(R \tilde{\sigma}_{N, v}, \tilde{u}_{N}-\tilde{u}_{P}\right)
\end{aligned}
$$

which yields to

$$
\left\|\tilde{u}_{N}(t)-\tilde{u}_{P}(t)\right\|_{V} \leq c\left(\left\|\tilde{f}_{N}(t)-\tilde{f}_{P}(t)\right\|_{V^{\prime}}+\left\|z_{N}(t)-z_{P}(t)\right\|_{\mathscr{H}}+\left\|\tilde{u}_{N}(t)-\tilde{u}_{P}(t)\right\|_{L^{2}\left(\Gamma_{3}\right)^{d}}^{1 / 2}\right) .
$$

On another hand we have

$$
z_{N}(t)-z_{P}(t)=\int_{0}^{t}\left(G\left(\bar{\sigma}_{N}(s), \varepsilon\left(\bar{u}_{N}(s)\right)\right)-G\left(\bar{\sigma}_{P}(s), \varepsilon\left(\bar{u}_{P}(s)\right)\right)\right) d s,
$$

thus

$$
\begin{aligned}
\left\|z_{N}(t)-z_{P}(t)\right\|_{\mathscr{H}} & \leq c\left(\int_{0}^{t}\left[\left\|\bar{\sigma}_{N}(s)-\bar{\sigma}_{P}(s)\right\|_{\mathscr{H}}+\left\|\bar{u}_{N}(s)-\bar{u}_{P}(s)\right\|_{V}\right] d s\right) \\
& \leq c\left(\frac{1}{N}+\int_{0}^{t}\left[\left\|\tilde{\sigma}_{N}(s)-\tilde{\sigma}_{P}(s)\right\|_{\mathscr{H}}+\left\|\tilde{u}_{N}(s)-\tilde{u}_{P}(s)\right\|_{V}\right] d s\right) .
\end{aligned}
$$

Combining (4.32) and (4.34), we obtain

$$
\begin{aligned}
\left\|\tilde{u}_{N}(t)-\tilde{u}_{P}(t)\right\|_{V} \leq c( & \frac{1}{N}+\left\|\tilde{f}_{N}(t)-\tilde{f}_{P}(t)\right\|_{V^{\prime}}+\left\|\tilde{u}_{N}(t)-\tilde{u}_{P}(t)\right\|_{L^{2}\left(\Gamma_{3}\right) d}^{1 / 2} \\
& \left.+\int_{0}^{t}\left[\left\|\tilde{\sigma}_{N}(s)-\tilde{\sigma}_{P}(s)\right\|_{\mathscr{H}}+\left\|\tilde{u}_{N}(s)-\tilde{u}_{P}(s)\right\|_{V}\right] d s\right) .
\end{aligned}
$$


Using (4.26), (4.35), and the definition of $\sigma_{N}$, we can easily prove

$$
\begin{aligned}
\| \tilde{\sigma}_{N}(t)- & \tilde{\sigma}_{P}(t) \|_{\mathscr{H}_{1}} \\
& \leq c\left(\frac{1}{N}+\int_{0}^{t}\left[\left\|\tilde{\sigma}_{N}(s)-\tilde{\sigma}_{P}(s)\right\|\left\|_{\mathscr{H}}+\right\| \tilde{u}_{N}(s)-\tilde{u}_{P}(s) \|_{V}\right] d s+\left\|\tilde{u}_{N}(t)-\tilde{u}_{P}(t)\right\|_{V}\right) .
\end{aligned}
$$

Writing

$$
F_{N, P}(t)=\left\|\tilde{\sigma}_{N}(t)-\tilde{\sigma}_{P}(t)\right\|_{\mathscr{H}_{1}}+\left\|\tilde{u}_{N}(t)-\tilde{u}_{P}(t)\right\|_{V},
$$

and mixing (4.35) and (4.36), we get

$$
F_{N, P}(t) \leq c\left(\frac{1}{N}+\left\|\tilde{f}_{N}(t)-\tilde{f}_{P}(t)\right\|_{V^{\prime}}+\left\|\tilde{u}_{N}(t)-\tilde{u}_{P}(t)\right\|_{L^{2}\left(\Gamma_{3}\right)^{d}}^{1 / 2}+\int_{0}^{t} F_{N, P}(s) d s\right) .
$$

As the sequence $\left(u_{N}\right)_{N}$ is bounded in $H^{1}([0, T], V)$, the sequence $\left(\left.u_{N}\right|_{\Gamma_{3}}\right)_{N}$ is relatively compact in $L^{2}\left([0, T], L^{2}\left(\Gamma_{3}\right)^{d}\right)$ (and in $C\left([0, T] ; L^{2}\left(\Gamma_{3}\right)^{d}\right)$ ). Moreover, as we have consider weakly convergent sequences, there is just one possible strong limit point of $\left(\left.u_{N}\right|_{\Gamma_{3}}\right)_{N}$ (which is of course the trace of $u$ ). We deduce that the sequence $\left(\left.u_{N}\right|_{\Gamma_{3}}\right)_{N}$ strongly converges in $C\left([0, T] ; L^{2}\left(\Gamma_{3}\right)^{d}\right)$ and $L^{2}\left([0, T], L^{2}\left(\Gamma_{3}\right)^{d}\right)$ and therefore is a Cauchy sequence in these spaces. Recalling the link between $\tilde{u}_{N}$ and $u_{N}$, we conclude that $\left(\left.\tilde{u}_{N}\right|_{\Gamma_{3}}\right)_{N}$ strongly converges in $L^{2}\left([0, T], L^{2}\left(\Gamma_{3}\right)^{d}\right)$ (and satisfies Cauchy criterium).

We write $G_{N, P}(t)=\int_{0}^{t} F_{N, P}^{2}(s) d s$. Let now $\rho>0$. Using (4.38) and for $N$ sufficiently large, we conclude that

$$
G_{N, P}(t) \leq \rho+c \int_{0}^{t} G_{N, P}(s) d s
$$

which proves with Gronwall's lemma that

$$
G_{N, P}(t) \leq \rho e^{c T}, \quad \forall t \in[0, T]
$$

The sequence $\left(\tilde{\mathcal{u}}_{N}, \tilde{\sigma}_{N}\right)_{N}$ is then a Cauchy sequence in $L^{2}([0, T] ; V) \times L^{2}\left([0, T] ; \mathscr{H}_{1}\right)$ and this ends the proof of Lemma 4.4 .

THE END OF THE PROOF OF LEMma 4.3. We prove that any (weak) limit point of $\left(u_{N}, \sigma_{N}\right)_{N}$ is solution of (1.5) and for this we will use system (4.26). Let then $(u, \sigma)$ be such a weak limit point in $H^{1}(0, T ; V) \times H^{1}\left(0, T ; \mathscr{H}_{1}\right)$.

Using Lemma 4.4, we easily have

$$
\begin{array}{ll}
u_{N}, \bar{u}_{N}, \tilde{u}_{N} \longrightarrow u & \text { strongly in } L^{2}(0, T ; V), \\
\sigma_{N}, \bar{\sigma}_{N}, \tilde{\sigma}_{N} \longrightarrow \sigma & \text { strongly in } L^{2}\left(0, T ; \mathscr{H}_{1}\right) .
\end{array}
$$


Using these convergences and the Lipschitz condition (1.8) on $G$, it is easy to prove that

$$
G\left(\bar{\sigma}_{N}, \varepsilon\left(\bar{u}_{N}\right)\right) \longrightarrow G(\sigma, \varepsilon(u)) \text { strongly in } L^{2}(0, T ; \mathscr{H}),
$$

hence (recalling (4.26a))

$$
\dot{\sigma}=\mathscr{E} \varepsilon(\dot{u})+G(\sigma, \varepsilon(u)) .
$$

Now we analyse the Signorini's condition. Let $\theta=\theta(t) \geq 0$ be any regular nonnegative function. Multiplying the third inequality of (4.26) by $\theta$, integrating over $(0, T)$, and use the strong convergence of $\tilde{\sigma}_{N}$ to $\sigma$ in $L^{2}\left(0, T ; \mathscr{H}_{1}\right)$, we pass to the limit in (4.26c) and obtain

$$
\left\langle\sigma_{v}, w_{v}-u_{v}\right\rangle \geq 0, \quad \forall w \in K .
$$

It still remains to study the second inequality in (4.26). We multiply it again by any nonnegative regular function $\theta=\theta(t)$, and integrate over $(0, T)$. The only term which is not straightforward is the term

$$
\int_{0}^{T} \theta(t) j\left(R\left(\tilde{\sigma}_{N v}\right)(t), \dot{u}_{N}(t)\right) d t=\mu \int_{0}^{T} \int_{\Gamma_{3}}\left|R\left(\theta \tilde{\sigma}_{N v}\right)(t)\right|\left|\dot{u}_{N \tau}(t)\right| d a d t .
$$

But we have

$$
\begin{aligned}
\int_{0}^{T} \int_{\Gamma_{3}}\left|R\left(\theta \tilde{\sigma}_{N v}\right)(t)\right|\left|\dot{u}_{N \tau}(t)\right| d a d t \geq & \int_{0}^{T} \int_{\Gamma_{3}}\left|R\left(\theta \sigma_{v}\right)(t)\right|\left|\dot{u}_{N \tau}(t)\right| d a d t \\
& -\int_{0}^{T} \int_{\Gamma_{3}}\left|\theta R\left(\tilde{\sigma}_{N v}(t)-\sigma_{v}(t)\right)\right|\left|\dot{u}_{N \tau}(t)\right| d a d t,
\end{aligned}
$$

hence

$$
\liminf _{N \rightarrow+\infty} \int_{0}^{T} \theta(t) j\left(R\left(\tilde{\sigma}_{N v}\right)(t), \dot{u}_{N}(t)\right) d t \geq \int_{0}^{T} \theta(t) j\left(R\left(\sigma_{v}\right)(t), \dot{u}(t)\right) d t .
$$

Assertions (4.43), (4.44), and (4.47) are sufficient in order to prove that the limit point $(u, \sigma)$ solve problem (1.5).

\section{REFERENCES}

[1] A. Amassad and C. Fabre, On the analysis of viscoelastic unilateral contact problem involving Coulomb friction law, to appear in J. Optimization Theory Appl.

[2] A. Amassad and M. Sofonea, Analysis of a quasistatic viscoplastic problem involving Tresca friction law, Discrete Contin. Dynam. Systems 4 (1998), no. 1, 55-72.

[3] P. Ballard, A counter-example to uniqueness in quasi-static elastic contact problems with small friction, Internat. J. Engrg. Sci. 37 (1999), no. 2, 163-178.

[4] M. Cocu, E. Pratt, and M. Raous, Formulation and approximation of quasistatic frictional contact, Internat. J. Engrg. Sci. 34 (1996), no. 7, 783-798.

[5] G. Duvaut and J.-L. Lions, Inequalities in Mechanics and Physics, Grundlehren der mathematischen Wissenschaften, vol. 219, Springer-Verlag, Berlin, 1976.

[6] N. Kikuchi and J. T. Oden, Contact Problems in Elasticity: A study of Variational Inequalities and Finite Element Methods, SIAM Studies in Applied Mathematics, vol. 8, SIAM, Pennsylvania, 1988.

[7] J. Nečas and I. Hlaváček, Mathematical Theory of Elastic and Elasto-Plastic Bodies: An Introduction, Studies in Applied Mechanics, vol. 3, Elsevier Scientific Publishing, Amsterdam, 1980. 
[8] M. Shillor and M. Sofonea, A quasistatic viscoelastic contact problem with friction, Internat. J. Engrg. Sci. 38 (2000), no. 14, 1517-1533.

AMINA AMASSAD: UNIVERSITÉ DE NICE-SOPHIA ANTIPOLIS, LABORATOIRE DE MATHÉMATIQUES J.A. DieudonnÉ, UMR 6621 du CNRS, PARC VALrose, 06108 Nice Cedex 2, France

E-mail address: amassad@math. unice.fr

CAROLINE FABRE: UNIVERSITÉ DE NiCE-SOPHIA ANTIPOlis, LABORATOIRE DE MATHÉMATIQUES J.A. Dieudonné, UMR 6621 du CNRS, PARC VAlrose, 06108 Nice Cedex 2, France

E-mail address: cfabre@math . unice. $\mathrm{fr}$ 


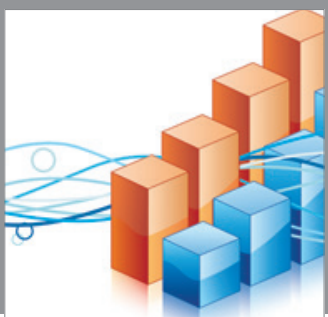

Advances in

Operations Research

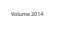

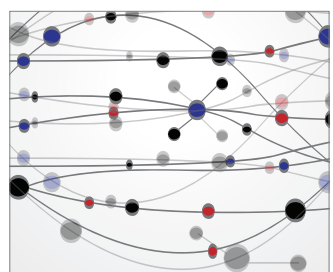

\section{The Scientific} World Journal
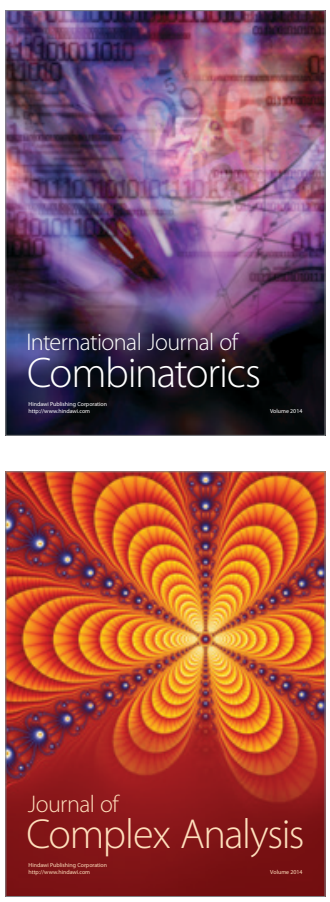

International Journal of

Mathematics and

Mathematical

Sciences
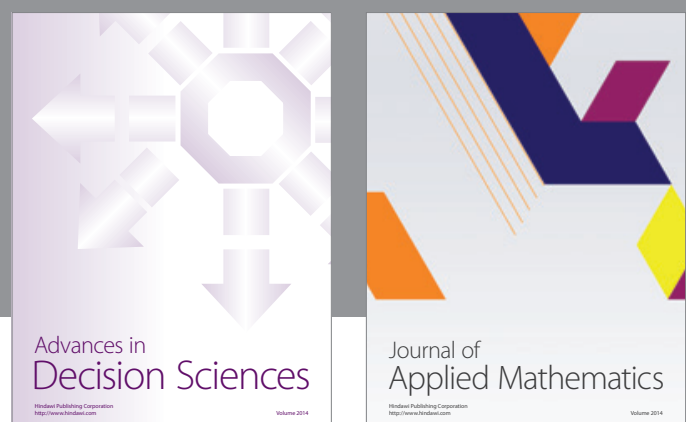

Journal of

Applied Mathematics
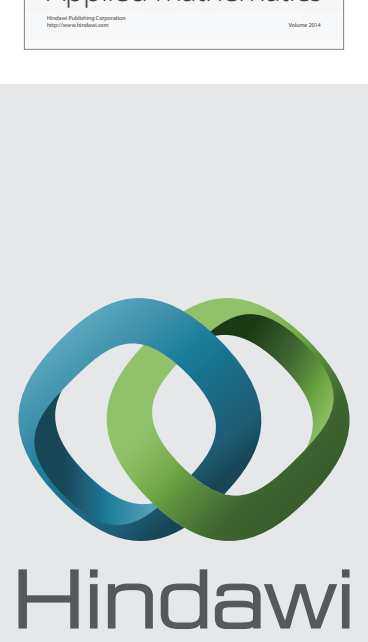

Submit your manuscripts at http://www.hindawi.com
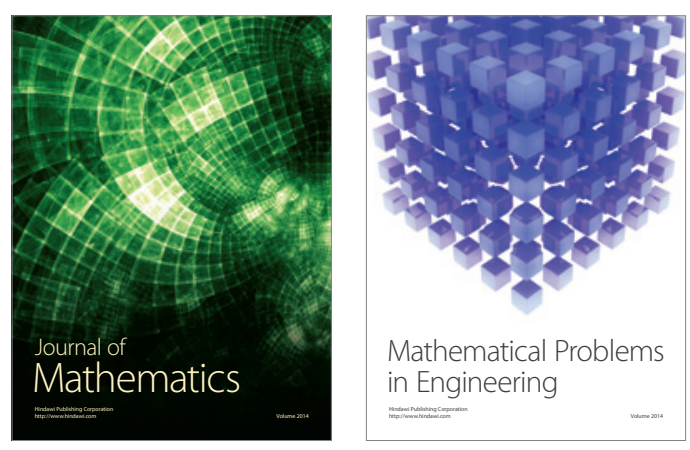

Mathematical Problems in Engineering
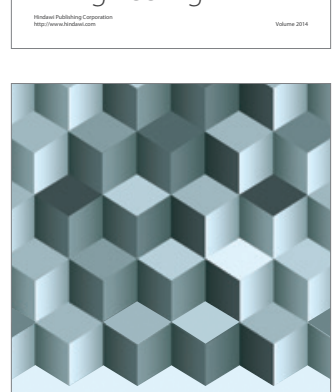

Journal of

Function Spaces
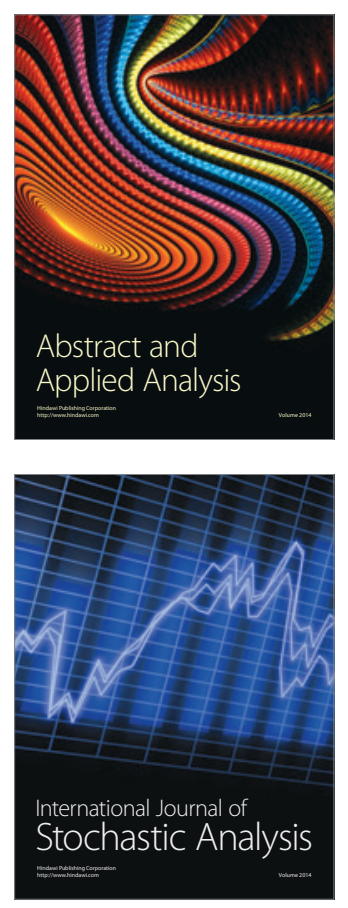

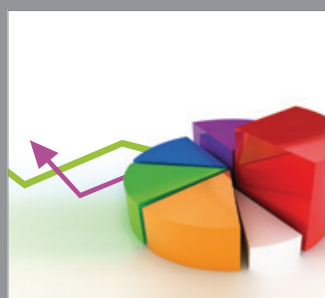

ournal of

Probability and Statistics

Promensencen
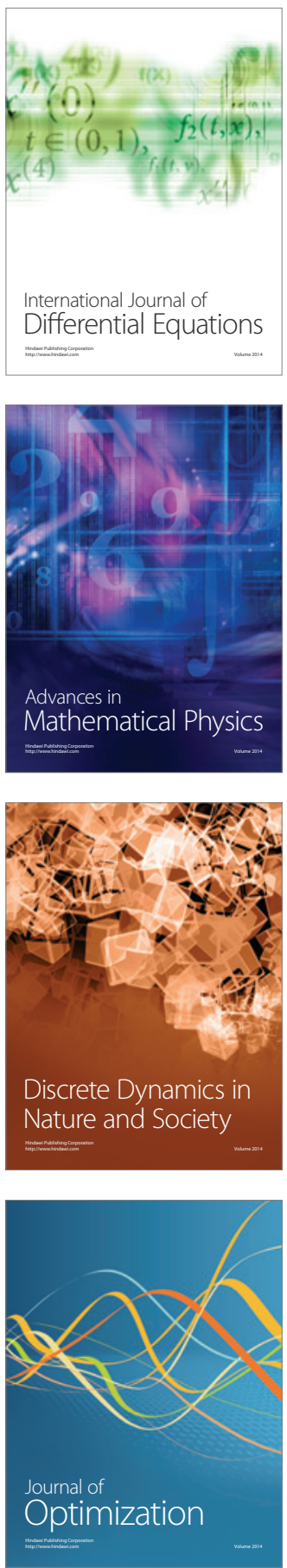